\title{
CFD Simulation of flow around angle of attack and sideslip angle vanes on a BAe Jetstream 3102 - Part 1
}

\author{
C J Bennett, N J Lawson, J E Gautrey, A Cooke \\ National Flying Laboratory Centre \\ School of Aerospace, Transport and Manufacturing \\ Cranfield University \\ C.J.Bennett@Cranfield.ac.uk
}

\begin{abstract}
CFD modelling techniques are exploited to investigate the local velocity field around angle of attack and sideslip angle sensors fitted to the nose of a modified BAe Jetstream 3102 small airliner. Analysis of the flow angularity at the vane locations has allowed the vanes response to varying flight conditions to be predicted and errors in the readings to be quantified. Subsequently, a more accurate calibration of the system is applied to the current configuration on the Jetstream, and a better understanding of the position error with respect to the vane locations is obtained.

The above aircraft was acquired by Cranfield University in 2003 with subsequent flow angle vane modifications taking place in 2005. The aircraft is currently in operation with the National Flying Laboratory Centre (NFLC) for research and demonstration purposes.
\end{abstract}

\section{INTRODUCTION}

The precise measurement of angle of attack and slideslip is critical in determining a number of key performance factors of any aircraft. Industry interest in angle of attack sensor design has intensified in recent years and is attributed to a spate of related accidents and incidents [1, 2]. Angle of Attack in particular is now seen as an essential parameter for safety and also efficiency, especially on commercial aircraft. It is well understood that high angles of attack can lead to stalling of the wings, however, sideslip is equally critical on the vertical control surfaces which determine the directional stability of the aircraft. Furthermore, flying with unnecessarily high angles of attack and sideslip increases drag, which in turn dramatically reduces fuel efficiency.

The accuracy of flow angle measurements is dependent upon the instruments chosen to gather the data, and also on the systematic and accurate calibration of this equipment.

In the comprehensive review of air-data systems and flow sensors [3], the authors noted three basic types of flow direction sensors: vanes, differential pressure sensors, and differential pressure probes. Vanes are small mass balanced winglets which align with the airflow during flight, allowing the angle of attack and sideslip angle to be measured depending on the axis of rotation to which they are mounted. Differential pressure systems on the other hand are less literal in that they calculate the angle of attack or sideslip angle by measuring the difference between two pressures at different locations. Vanes are preferred in many cases since, despite being a relatively inexpensive modification to any aircraft, a high level of accuracy can be obtained if accurately calibrated. It can be seen from the photograph in Fig 1 that the Jetstream 31 in question has undergone the flow vanes modification.

Published by Elsevier. This is the Author Accepted Manuscript issued with: Creative Commons Attribution Non-Commercial No Derivatives License (CC:BY:NC:ND 4.0). The final published version (version of record) is available online at DOI:10.1016/j.ast.2017.03.015. Please refer to any applicable publisher terms of use. 


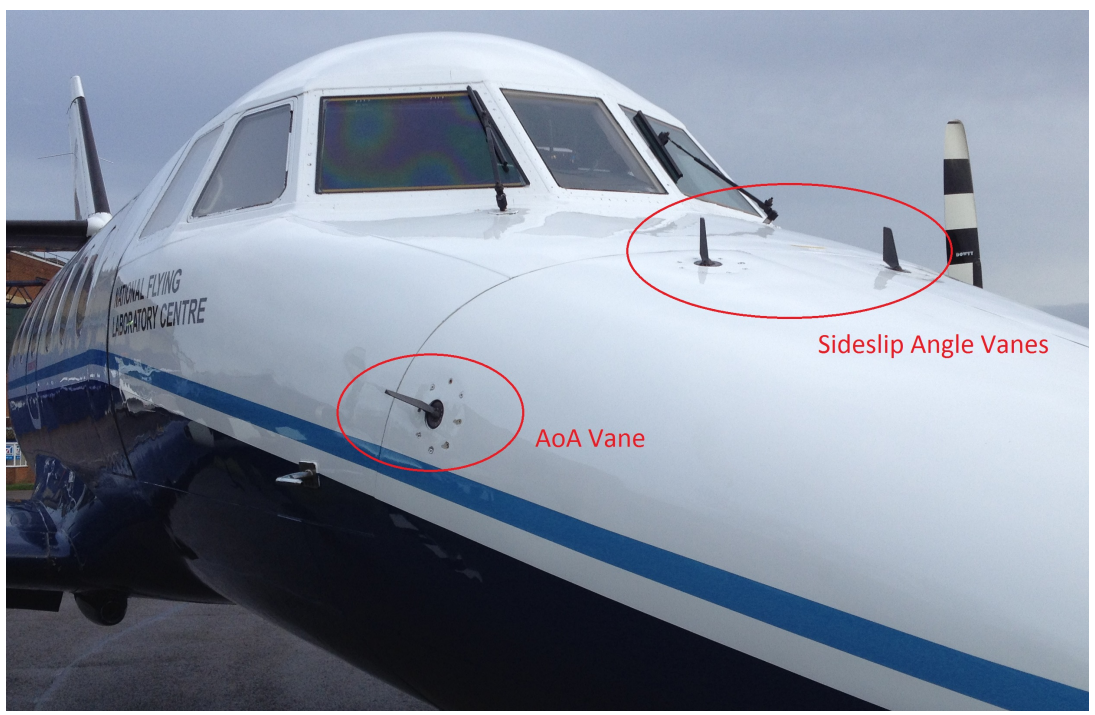

Figure 1: Angle of attack (AoA) vane and sideslip angle vane placement on the BAe Jetstream 3102

It must be noted that vanes of this type are somewhat universal in that they are not designed for a specific aircraft, instead they are designed to be effective for a wider class of aircraft. Furthermore, the vanes are not accompanied with drawings of recommended positions for installation on a specific aircraft, so it is the users responsibility to decide on an effective location for the vanes and to calibrate the readings accurately.

The inherent error of flow angle vanes is due to the difference between the local flow angularity and the true freestream values and is called the 'position error'. The position error is difficult to calculate due the large number of contributing factors. As a result, analysis techniques have been developed and refined by aerospace research groups since the 1970's. Many publications have proposed novel ways in which to calibrate flow vanes to eliminate these errors, but it is the large number of factors which are present in any given flight condition which make this problem very type specific, and therefore generic guidelines for calibration are difficult to establish. The following paper will demonstrate that advanced techniques in computational fluid dynamics (CFD) provides an alternative method for overcoming this calibration issue.

It is worth noting that the majority of the literature on this topic deals with vanes mounted to a boom or probe usually extended out from the nose. In the case of a boom, the vanes are exposed to near freestream conditions. In the nose region, the flow field has a thin, attached, boundary layer and therefore the required height/length of the probe is minimised. The benefits and drawbacks of this set-up compared to the nose mounted vanes, as seen on the Jetstream, are discussed in detail later. The literature regarding nose boom mounted vanes is of course relevant here and should be understood and applied to this investigation, however, the relative importance of the factors leading to error are somewhat different.

In this study, CFD simulations will enable us to visualize the local flow around the sensors. By refining the mesh density around the vane positions, and also by running a comprehensive matrix of flight conditions, it will be shown that the CFD simulations will provide reliable prediction of the flow and hence the error characteristics of the vanes.

The following section summarizes a number of factors which can lead to errors in the data recorded by angle of attack and sideslip angle sensors. A discussion of potential improvements to 
calibration and mounting locations for the sensors is presented later using a validated CFD model as a tool for design refinement.

\section{Angle of Attack And Sideslip Angle Sensor Errors}

There are five main considerations when analysing the position error of flow vanes: air flow effects, vane float, structural deformation, installation errors, and for higher mach number flight, shock wave effects. A summary of these concepts, in the order in which they are predicted to have most effect on the flow vanes, is given below. A discussion on how such errors may be addressed is also provided.

Firstly, it is critical to understand how the airflow direction in the region around the flow angle sensors is disturbed from the freestream value by the physical structure of the aircraft. The air flow must deviate from freestream conditions to pass over and around the body in flight. This change in airflow direction, if occurring in the region of the flow vanes, may cause under- or over-reading of angle of attack and sideslip angle. Furthermore, if the flow in these regions becomes separated or turbulent, the vanes may exhibit unpredictable and erratic behaviour.

Considering the nose mounted location of the flow vanes on the Jetstream, the airflow may be affected in this region in a number of ways. As mentioned above, the airflow passing over the nose of the aircraft close to the surface may differ considerably with respect to the freestream values. This effect is expected to be greater for higher angles of attack and sideslip, and may also reach a point of flow separation and turbulence in extreme cases. For example, in the current configuration, Fig. 1. if the Jetstream is flying with a positive angle of attack and negative sideslip angle the angle of attack sensor may be obscured from the oncoming freestream. Depending on the Reynolds number, the flow in this obscured region may detach and form a turbulent wake.

Propellers can also have a major effect on the airflow around the aircraft. It is well understood that a rotating propeller induces a helical slipstream over the aircraft. This rotating slipstream imposes a side force on the fin, rudder, and rear fuselage leading to an undesired yawing effect which must be counteracted by the pilot using the rudder. This means that in maintaining straight and level flight, the aircraft is travelling with a constant sideslip. In the case of the Jetstream, a twin-turboprop aircraft, both propellers rotate in the same direction increasing the above effect. Although the induced rotating slipstream will not directly affect the flow angle vanes as they are mounted upstream of the propellers, it is important to understand the inherent sideslip effects of propeller aircraft for comparison with flight test data and calibration of the sensors. For this study, upsteam flow disturbances induced by the propellers will be assumed negligible.

For subsonic flows, flow disturbances caused by the aircraft travelling through the air propagate radially. These disturbances contribute to the overall disparity between the local flow and the freestream but, due to the range of Mach numbers discussed here, are not considered as important as the effects described above.

Secondly, manufacturing defects or damage can lead to the vanes performing differently depending on their orientation. This effect, referred to in the literature as 'vane float', is a very minor consideration compared to that of the air flow effects. However, it is much easier to quantify with experimentation, details of which are given in the next section.

Thirdly, structural deformation may cause measurement inaccuracy of the flow vanes [4]. This effect is more pronounced when the vanes are mounted on booms or probes, as these structures are more susceptible to bending under load in flight. However, similar effects may also occur on

\footnotetext{
${ }^{1}$ It is convention for angle of attack to be measured as positive for nose-up flight and for sideslip to be measured positive when the relative wind approaches from the right of the aircraft (or the nose is pointing left of the direction of flight).
} 
airframe panels to which the vanes are mounted. Furthermore, at certain velocities vibrational frequencies of a particular boom or body panel may lead to noise in the sensed data. Again, these effects are more pronounced on other parts of the airframe, for example the wings, as they are more vulnerable to flexing during flight.

Lastly, it is reasonable to assume that the vanes have been installed accurately with respect to the drawing locations and are correctly set-up for use.

Another consideration which must be discussed is that of upwash and downwash. Depending on the flight conditions, upwash and downwash effects can subject the wing to a local effective angle of attack different to that defined by the difference between the flight path and the wing reference line. Obviously this effect can not be captured by vanes mounted on the nose. However, it is expected to be negligible except for extreme angles of attack, close to stall conditions.

Finally, although beyond the scope of this paper due to the Jetstream's capabilities, it is worth mentioning for completeness that for transonic and supersonic flows shock wave propagation can lead to further unexpected behaviour from the sensors [4].

In recognition of the considerations outlined above, the following section discusses calibration methods aimed at eliminating or reducing flow angle sensor errors.

\section{Calibration}

This study will rely on results obtained from CFD and comparisons with pre-existing flight test data, due to the constraints of cost and availability. The analysis will assume that the effects due to vane float and structural deformation are negligible compared to that of the airflow effects. To analyse the local flow around the sensors, CFD simulations will be performed for varying angles of attack and sideslip. Once the local flow around the vanes has been predicted, sufficiently validated, and a profile of their behaviour is developed for a range of flight conditions, calibration of the vanes can be performed.

The use of CFD and flight test data however, are just two examples of the calibration methods available. The literature outlines four main types of calibration tests: theoretical calculations and computer simulations, wind tunnel analysis, in flight testing, and structural strength experiments. Clearly, if a range of different tests can be performed, the accuracy of the results will be improved. A brief explanation of each calibration method is given below.

A theory for predicting upwash angles induced by propeller rotation was pioneered by Yaggy and Rogallo [5, 6] in the 1950's. The so-called Yaggy-Rogallo method was successfully used by NASA flight test research facilities for many years ${ }^{2}$, at least until Rawlings III published a paper [7] attempting to combine Yaggy and Rogallo's theories to present a definitive user guide for the method. Rawlings III [7] also presented example FORTRAN computer codes to aid the numerical integration required for future exploiters of the method. Essentially, the method involves separating the airframe in question into non-lifting surfaces and thin aerofoil sections. Then, the non-lifting sections are split into a series of bodies of rotation based on an approximate radius, and the upwash angle for each segment calculated using an integral equation. The total upwash angle is found by summing all individual elements. The thin aerofoil sections are treated similarly. The method is valid for low subsonic flows and for angles of attack and sideslip less than $15^{\circ}$, within the scope of this paper.

Secondly, in the case of wind tunnel testing, it is important for the user to consider general wind tunnel characteristics before dealing with the specific problem requirements. For example, should the wind tunnel not obtain the required Reynolds number, trip strips may be required in

\footnotetext{
${ }^{2}$ The method's current status in flight test and academic circles is unknown
} 
order to artificially replicate the development of the turbulent boundary layer. Furthermore, the test model should have sufficient space around it as not to be affected by model blockage effects.

Although wind tunnel testing is unable to incorporate the effects of propeller upwash and other propulsion effects such as the vibration of an engine as discussed by Lawford and Nippress [3], the airflow over the body can be accurately replicated and the flow field visualized. Furthermore, it is possible to isolate conditions which produce individual errors.

In the detailed wind tunnel investigation [4], the author presented error characteristics for both angle of attack and slidslip angle vanes mounted to a boom for a range of Mach numbers. The full scale boom with attached flow vanes was used for the wind tunnel analysis. In order to calibrate the equipment, a laser was mounted to the boom and aimed at a benchmark on the wind tunnel wall. These readings gave the true angles of attack and sideslip in each test case, and were compared to the sensor readings. The accuracy, in general, was better for lower Mach numbers and lower angles of attack and sideslip, but deteriorated as these values increased. The data showed a notable rise in error as the Mach number approached 1, and so it was concluded that shock wave interaction was the main contributor to the error for the range of Mach numbers considered. However, for the scope of this paper where a narrow range of subsonic Mach numbers are considered, it is seen that the error remained relatively small and increased approximately linearly for this range.

The third branch of calibration methods to consider is in flight testing. Although causes of particular errors are difficult to isolate in flight, it is important to correlate predictions with actual flight data. Lawford and Nippress [3] explored in detail the full range of flight calibration methods and explained that data could be gathered from three different states of flying: steady state, quasi-steady state, and dynamic manoeuvres. For the purpose of this paper however, our attention will be focused on steady state calibrations due to our access to a substantial body of historical data from straight and level flight and steady heading slideslip flight tests.

The final type of calibration test to consider concerns the structural deformation or bending of the equipment under load. These experiments are perhaps the simplest to perform and the results easiest to interpret as the cause of the error is isolated from other sources. Sakamoto [4] performed a simple test to measure the deflection of a nose boom. An aerodynamic force was applied on the boom in an upward direction and the bending effect was determined to be negligible. As discussed above, a nose boom is expected to experience greater deformation under load in flight than actual airframe panels due to it's high aspect ratio, and so this effect will not be considered here, although it is acknowledged that vibrational modes of the body panels in flight are often attributed to noise in the sensed flight test data.

\section{CFD MOdEL}

A full Jetstream geometry definition, originally developed by Vessot [8] following a full aircraft scan by Parker [9], was modified in ANSYS ICEM CFD to incorporate additional surfaces on the nose of the aircraft to represent the flow vane locations, see Fig 2. The modifications were applied to the model in accordance with the original scale drawings. Before meshing, the topology was checked and cleaned to ensure the integrity and closure of all surfaces. It should be noted, that due to the wing twist of the Jetstream, the angle of attack in all subsequent analysis is measured with respect to the fuselage reference line and the flight path of the aircraft or relative wind. The fuselage reference line is parallel to the centre section of the fuselage and is applicable to both the CFD data obtained via the model described in this section and also the flight test angle of attack data in a later section.

A series of hybrid meshes were generated using the Octree volume meshing algorithm in 

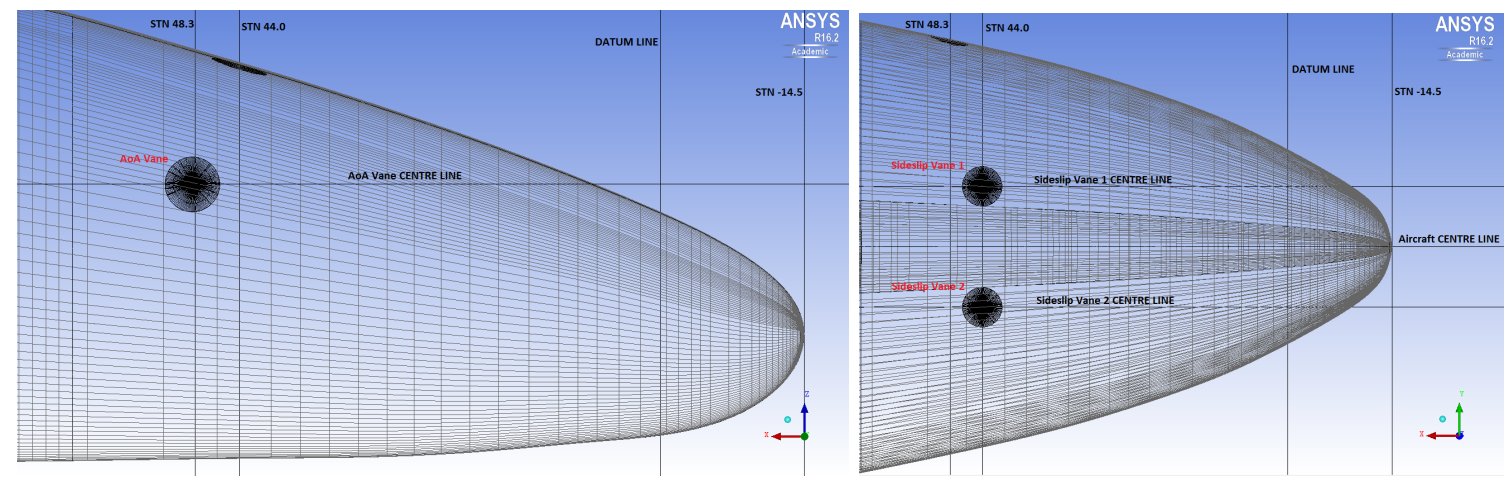

Figure 2: Angle of attack and sideslip angle vane modification to full Jetstream model in ANSYS ICEM CFD. Station numbers (STN) are given in inches in accordance with the original scale drawings for the modification. The sideslip vane centre lines are \pm 8.6 inches from the aircraft centre line in the $y$-direction, and the angle of attack vane centre line is approximately \pm 12.74 inches from the top of the nose in the $z$-direction.
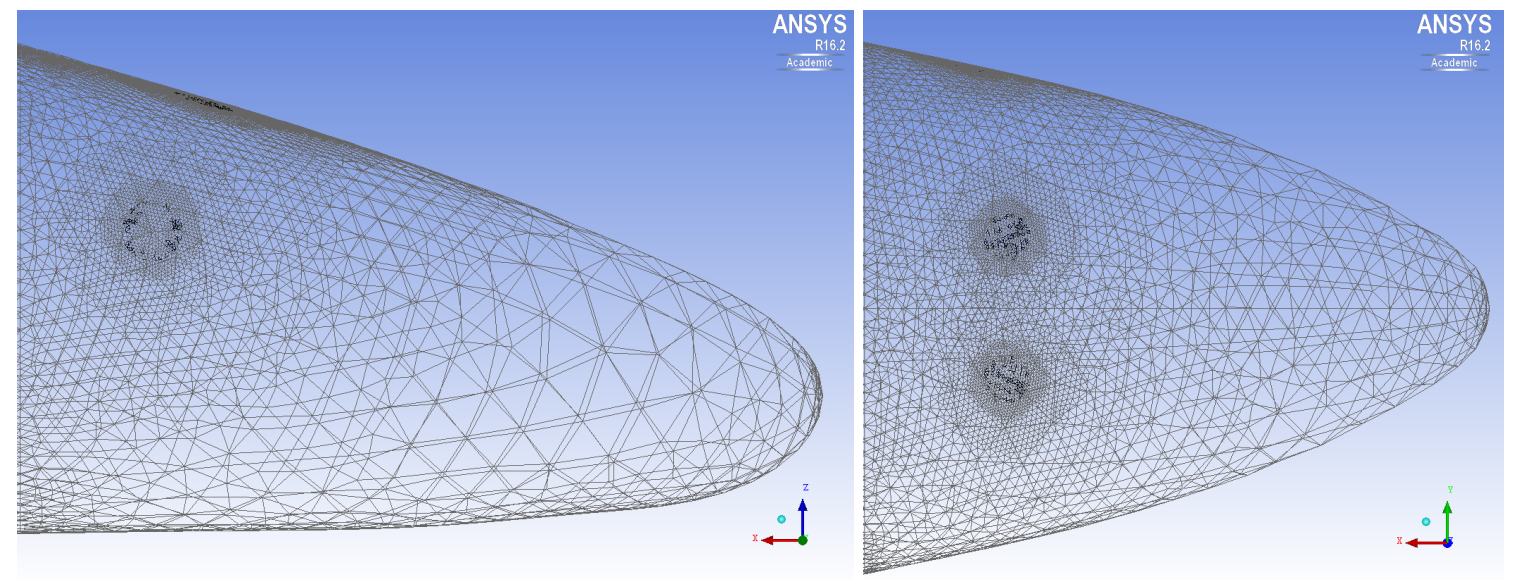

Figure 3: Refinement of the mesh density around the nose and flow angle vanes.

conjunction with the prism layer generator. It is assumed for the purposes of the simulations conducted here that the boundary layer can be adequately described by the log law and hence 10 prism layers are specified at a growth ratio of 1.2 starting from an initial cell height of $0.2 \mathrm{~mm}$. The range of typical Reynolds numbers, $\operatorname{Re}=4.85-9.94 \times 10^{6}$, based on the wing chord of $1.717 \mathrm{~m}$, and corresponding Mach number range, $\mathrm{M}=0.15-0.30$, yields a non-dimensional wall distance range of $y^{+}=21-41$. The differences in Reynolds number over this range is seen to have negligible effect on the vane forces. To increase the accuracy of the simulations the mesh densities were significantly increased in the region around the nose and flow angle vanes, see Fig 3

Three separate meshes, coarse, medium and fine, were generated with increasing densities on and around the nose in order to compare the accuracy of the solution with regard to the additional computational expense. Table 1 summarizes the number of elements for each mesh.

Each mesh was analysed to ensure that an acceptable mesh quality was achieved. Firstly, a global Laplace smoothing algorithm was applied to each mesh, followed by analysis of the mesh quality using the quality histogram tool. For all mesh densities, less than $0.2 \%$ of the total number 


\begin{tabular}{|l|c|c|c|}
\hline Mesh & Total Elements $\left(\times 10^{6}\right)$ & $\begin{array}{c}\text { Nose Element } \\
\text { Max Size }(\mathrm{mm})\end{array}$ & $\begin{array}{c}\text { Vane Element } \\
\text { Max Size }(\mathrm{mm})\end{array}$ \\
\hline Coarse & 8.77 & 600 & 30 \\
Medium & 9.03 & 400 & 10 \\
Fine & 9.44 & 200 & 5 \\
\hline
\end{tabular}

Table 1: Summary of mesh element count and maximum element sizes

\begin{tabular}{|l|l|c|}
\hline Mesh & Vane & \% Difference in Force with respect to Fine Mesh \\
\hline Coarse & Angle of Attack & 0.0191 \\
& Sideslip & 0.0185 \\
Medium & Angle of Attack & 0.0076 \\
& Sideslip & 0.0121 \\
\hline
\end{tabular}

Table 2: Summary of percentage difference of force exerted on vanes due to reduced accuracy of coarse and medium mesh densities

of cells attained a quality of 0.1 or less, and approximately $0.5 \%$ of cells attained a quality of 0.3 or less. The medium and fine meshes offered improvements on the quality although not significant.

Velocity profiles plotted along the length of both angle of attack and sideslip angle vanes were analysed to decide the most suitable mesh density and turbulence model with which to proceed. It is seen from the example given in Fig. 4 that all mesh densities give near identical velocity profiles only differing marginally in the region of the boundary layer as expected. Furthermore, force calculations based on the approximate area of the vanes show that the discrepancy in flow velocity in the coarse and medium mesh cases compared to that in the fine mesh case yields a negligible difference, a summary of which is given in Table 2 Therefore considering the velocity profiles, the increase in mesh density is not justified.

Further analysis of the mesh densities was undertaken in the form of surface pressure coefficient $\left(C_{p}\right)$ plots. Surface lines were drawn on the aircraft nose bisecting the angle of attack vane or one of the sideslip vanes and $C_{p}$ was plotted along these lines.

In the cases shown in Fig. 5 for example, it is seen that the differences in mesh density more noticeably affect the $C_{p}$ plots, particularly in the regions of the vanes themselves: approximately located at $0.12-0.14$ in the $x$-axis for the angle of attack vane, and approximately at $0.11-0.13$ in the $\mathrm{x}$-axis for the sideslip vane, see Fig. 5. Here, the jump in mesh density between the nose and the vanes causes noise in the data, particularly in the coarse and fine cases, and may also be discontinuous. It is therefore concluded that the medium mesh is most suitable based on the $\mathrm{C}_{p}$ plots.

For further verification and validation, simulations were analysed for a variety of turbulence models: the one-equation Spalart-Allmaras (S-A) model, the two-equation $k-\varepsilon$ realizable model, and the $k-\varepsilon$ SST model. The medium mesh density was used as an example to compare the variation in turbulence models.

Fig. 6 shows an example velocity profile plotted along the length of the angle of attack vane. The different turbulence models affect the velocity profiles in the boundary layer as expected, see $x=0$ to $x \approx 1.25 \times 10^{-3}$, however the maximum difference in velocity is only around $10 \%$ in this region, leading to negligible applied force discrepancy summarised in Table 3 It is seen that the more complex 2-equation models do not considerably affect the applied force to the turning vanes 


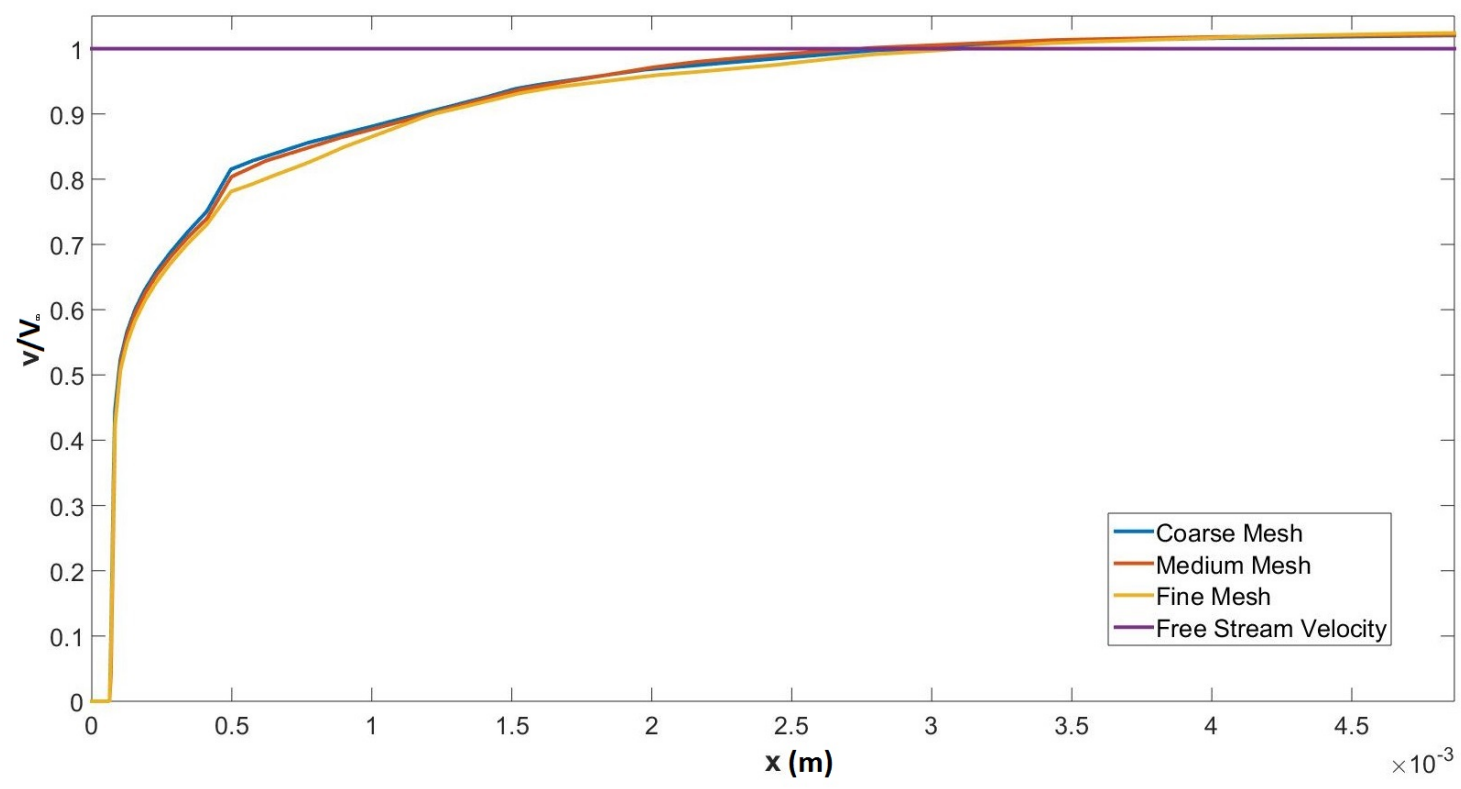

Figure 4: An example velocity profile comparing three mesh densities for the angle of attack vane in the case AoA=9.4 and $S S=8^{\circ}$. Velocity, $v$, is non-dimensionalised with respect to the freestream velocity, $v=64.31 \mathrm{~ms}^{-1}$. The distance from the surface, $x$, is non-dimensionalised with respect to the fuselage length, $l=14.37 \mathrm{~m}$ and the range given corresponds approximately to the height of the vane.

\begin{tabular}{|l|l|c|}
\hline Turbulence Model & Vane & \% Difference in Force with Respect to S-A Model \\
\hline k- $\varepsilon$ & Angle of Attack & 2.51 \\
& Sideslip & 1.96 \\
SST & Angle of Attack & 0.73 \\
& Sideslip & 0.78 \\
\hline
\end{tabular}

Table 3: Summary of percentage difference of force exerted on vanes due to increased accuracy of $k$-E and SST turbulence models.

and hence the added computational expense to each simulation is not justified.

Furthermore, Fig. 7 shows that the two 2-equation models do not significantly change the $\mathrm{C}_{p}$ plots compared to the 1-equation S-A model. Therefore the S-A turbulence model and the medium mesh were chosen to carry out the full matrix of simulations.

The model was solved in Fluent using a Reynolds averaged Navier-Stokes (RANS) finite volume implicit solution with second order upwind accuracy. A steady state, density based solution was also specified. The density of the air inside the fluid volume was treated as an ideal gas and the viscosity described by the Sutherland law. All airframe surfaces were specified with wall type boundary conditions and the domain boundaries treated as the pressure far-field. The reference values were taken from accurate aircraft data in conjunction with typical flight test conditions, see Table 4. The lift and drag coefficients were monitored to analyse the convergence of the solutions. A residual of less than $10^{-5}$ determines satisfactory convergence for this type of application [10], and based on this criteria, convergence of the solution was attained in 4000-5000 iterations. 
1)

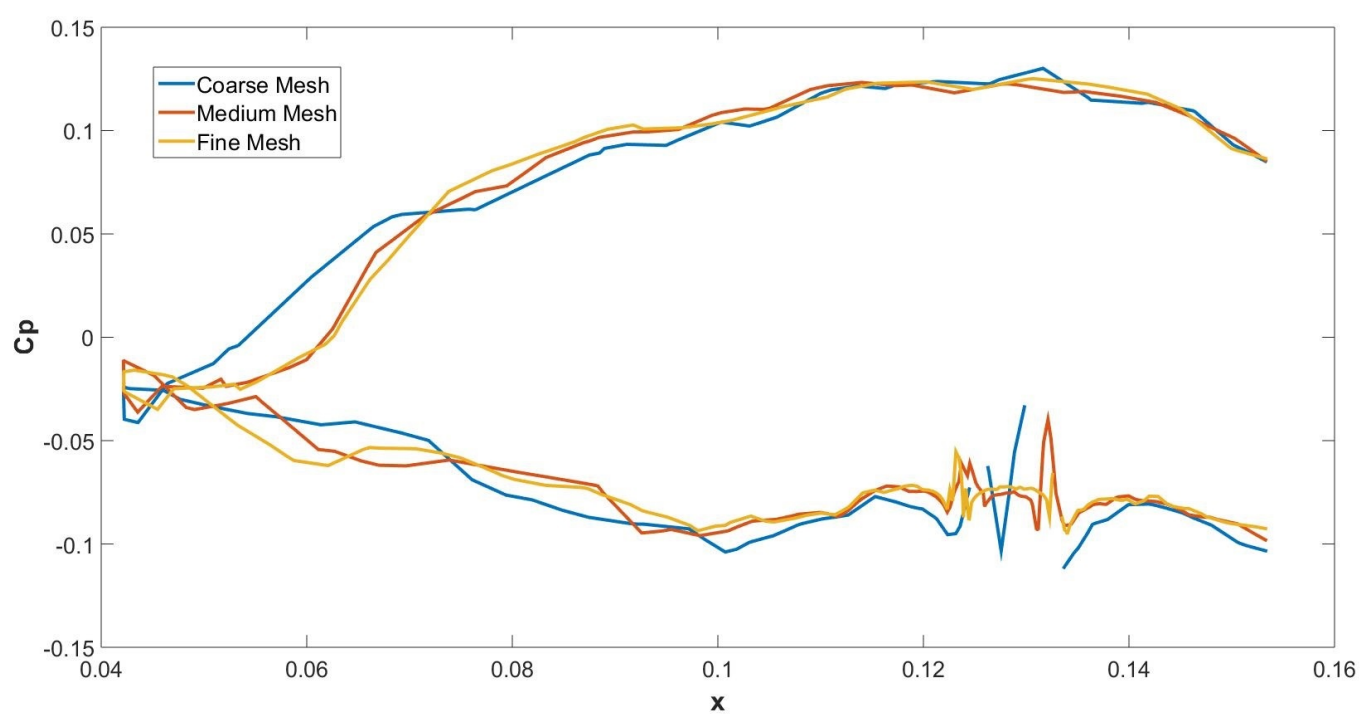

2)

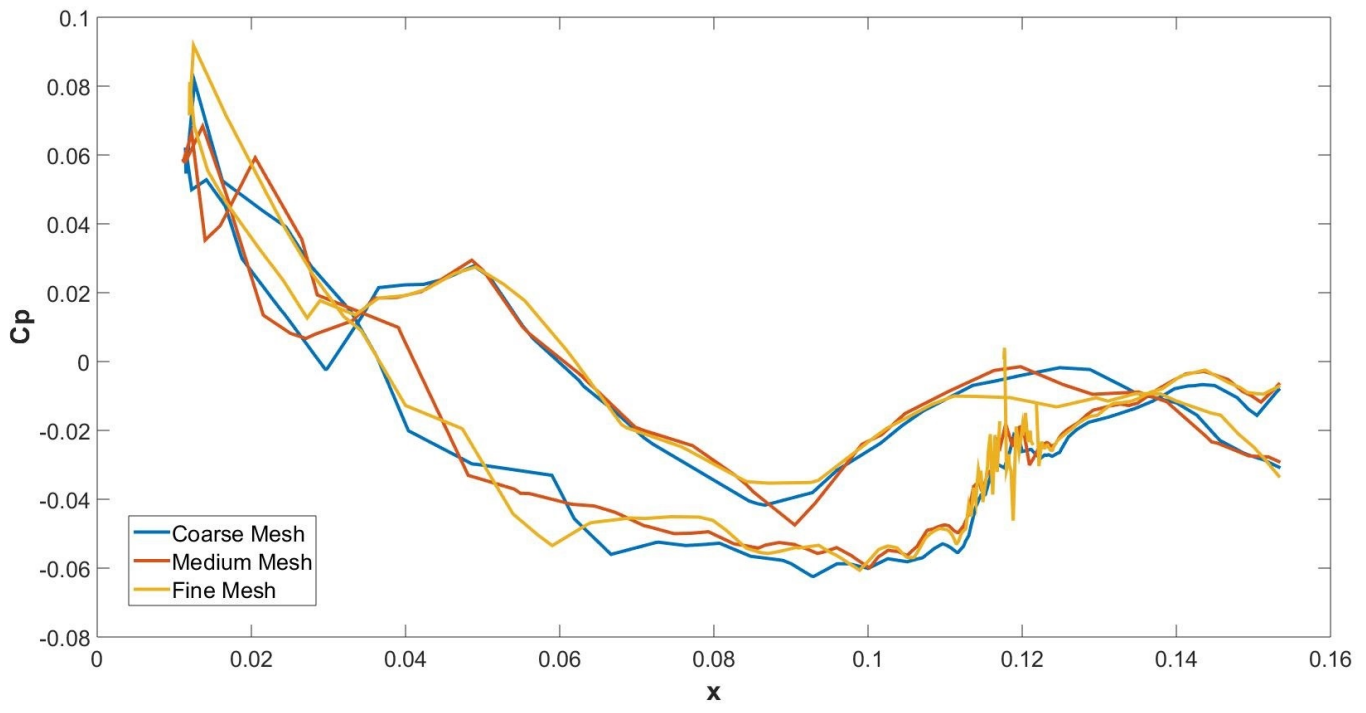

Figure 5: Comparison of the mesh densities with $C_{p}$ plots of 1) angle of attack vane, and 2) sideslip angle vane for the case $A o A=9.4^{\circ}$ and $S S=8^{\circ}$. 


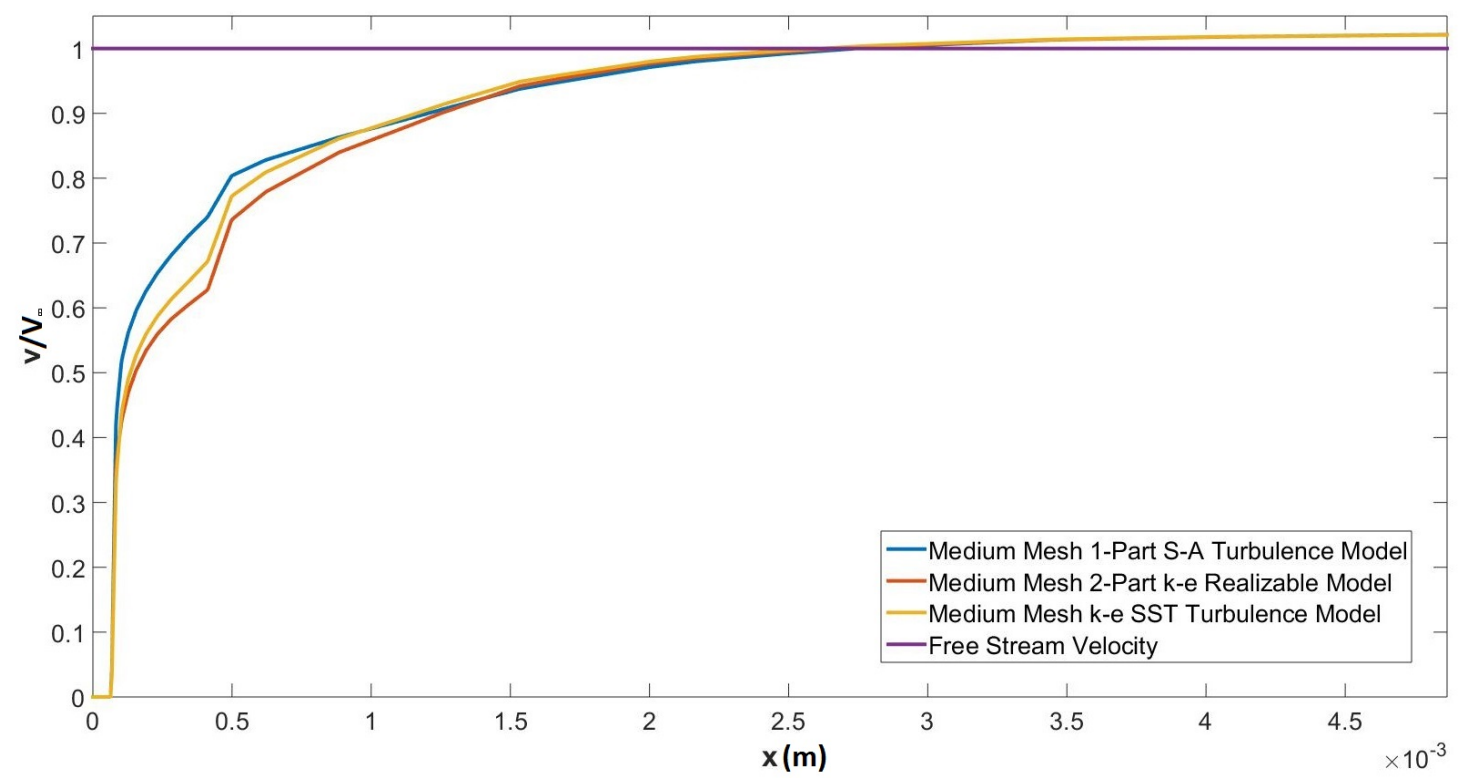

Figure 6: Comparison of the turbulence models with velocity profile at the angle of attack vane for the case AoA=9.4 and $S S=8^{\circ}$.

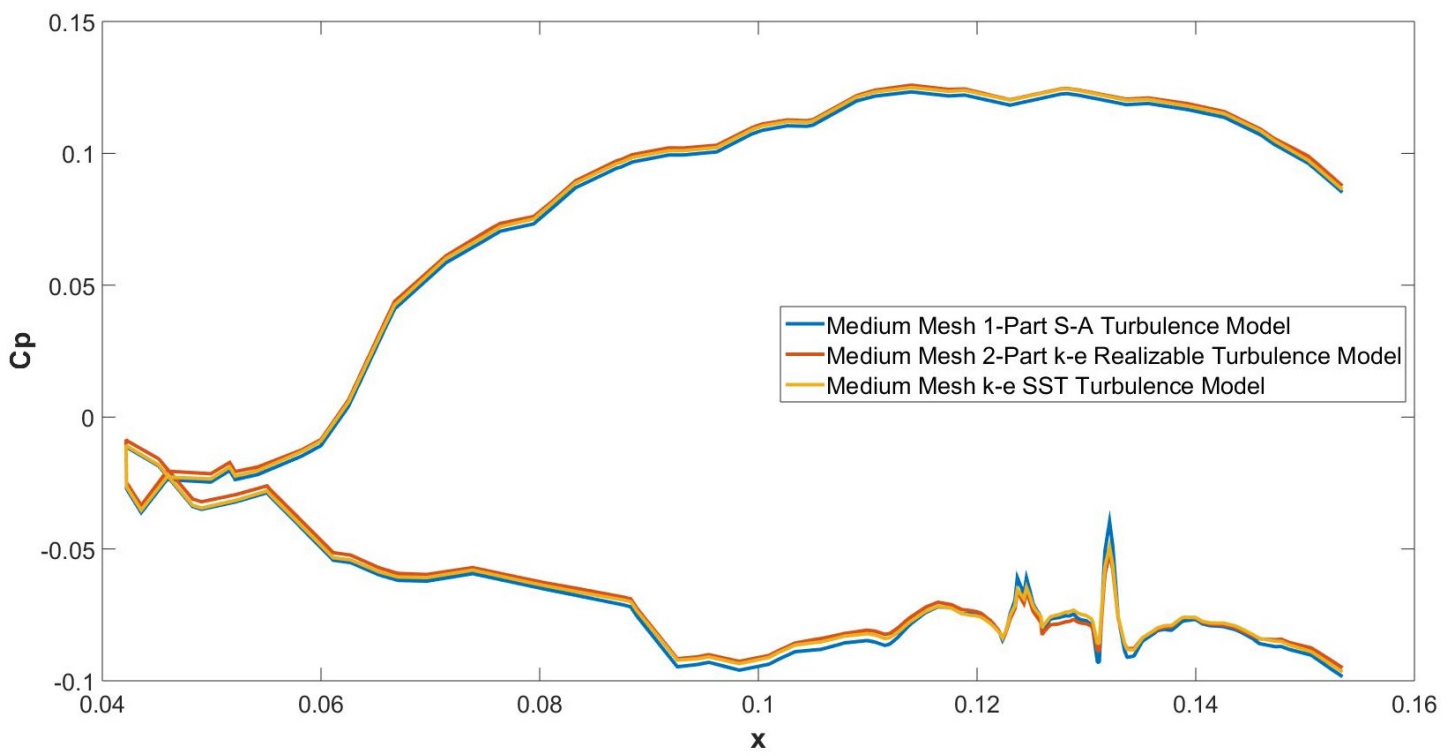

Figure 7: Comparison of the turbulence models with $C_{p}$ plot for angle of attack vane for the case AoA $=9.4^{\circ}$ and $S S=8^{\circ}$. 


\begin{tabular}{|l|l|}
\hline Area (wing) $\left(\mathrm{m}^{2}\right)$ & 25.085 \\
Density $\left(\mathrm{kg} / \mathrm{m}^{3}\right)$ & 0.9936 \\
Enthalpy $(\mathrm{j} / \mathrm{kg})$ & 280906.5 \\
Length $(\mathrm{mean}$ chord) $(\mathrm{m})$ & 1.717 \\
Pressure $(\mathrm{Pa})$ & 78185 \\
Temperature $(\mathrm{K})$ & 274.15 \\
Velocity $(\mathrm{m} / \mathrm{s})$ & $64.31-100.3$ \\
Viscosity $(\mathrm{kg} / \mathrm{m}-\mathrm{s})$ & $1.721 \times 10^{-5}$ \\
Ratio of Specific Heats & 1.4 \\
\hline Operating Pressure $(\mathrm{Pa})$ & 78185 \\
\hline
\end{tabular}

Table 4: Table of reference values used for Fluent solver

\section{CFD Results ANd AnAlysis}

CFD simulations were firstly analysed to determine the correlation of lift and drag coefficients with the flight test data. The coefficients in all cases were calculated using the Fluent force report function. It was seen that, for zero sideslip cases, $C_{D}$ was underestimated by $22 \%$ for low angles of attack, ranging to $32 \%$ for high angles of attack. This result can be attributed to the simplification of the Jetstream model to not include the propellers, flaps, aerials and other protuberances. $C_{L}$ on the other hand, was underestimated by between $16-20 \%$ compared with the flight test data. The shortfalls in lift when comparing the CFD results to flight test data can be attributed to the propeller induced slipstream effects as discussed in [11, 12].

The differences in flow angles locally around the vanes as compared to the freestream are attributed to the effect of the aircraft nose of the local flow field around it as discussed previously. The example streamline plots in Fig. 8 for high positive and negative sideslip angles at the same angle of attack, show how the airflow deflects around the nose of the aircraft influencing the directional response of the vane. It is seen that the streamline angle passing over the angle of attack vane location is considerably different in each case despite the fact that the freestream angle of attack is equal. The following analysis attempts to quantify not only how the raw vane readings can be interpreted to represent the true (or freestream) flow direction, but also illustrates how the aircraft nose influences the local flow direction around the vanes for the full range of in flight motion.

In order to understand the behaviour of the flow angle vanes in each simulation, a detailed investigation of the local flow in these areas was undertaken. Firstly, vane centre lines were drawn in CFD-Post perpendicular to the aircraft nose to an accurate length in accordance to the scale drawings for the vane modifications. Secondly, directional velocity components $u, v$ and $w$ as well as total velocity $V$ were plotted at 100 equally spaced intervals along the angle of attack and two sideslip vane centre lines. The local angle of attack and sideslip at each of these points can be calculated, if the bank angle of the aircraft is assumed to be zero (see Appendix (A) for derivation), thus

$$
\begin{gathered}
\alpha=\tan ^{-1}\left(\frac{w}{u}\right), \\
\beta=\sin ^{-1}\left(\frac{v}{|V|}\right) .
\end{gathered}
$$

To determine the relative contribution of the flow angle at these individual points to the overall directional response of the vanes, the flow angle data was weighted based on the area of the 

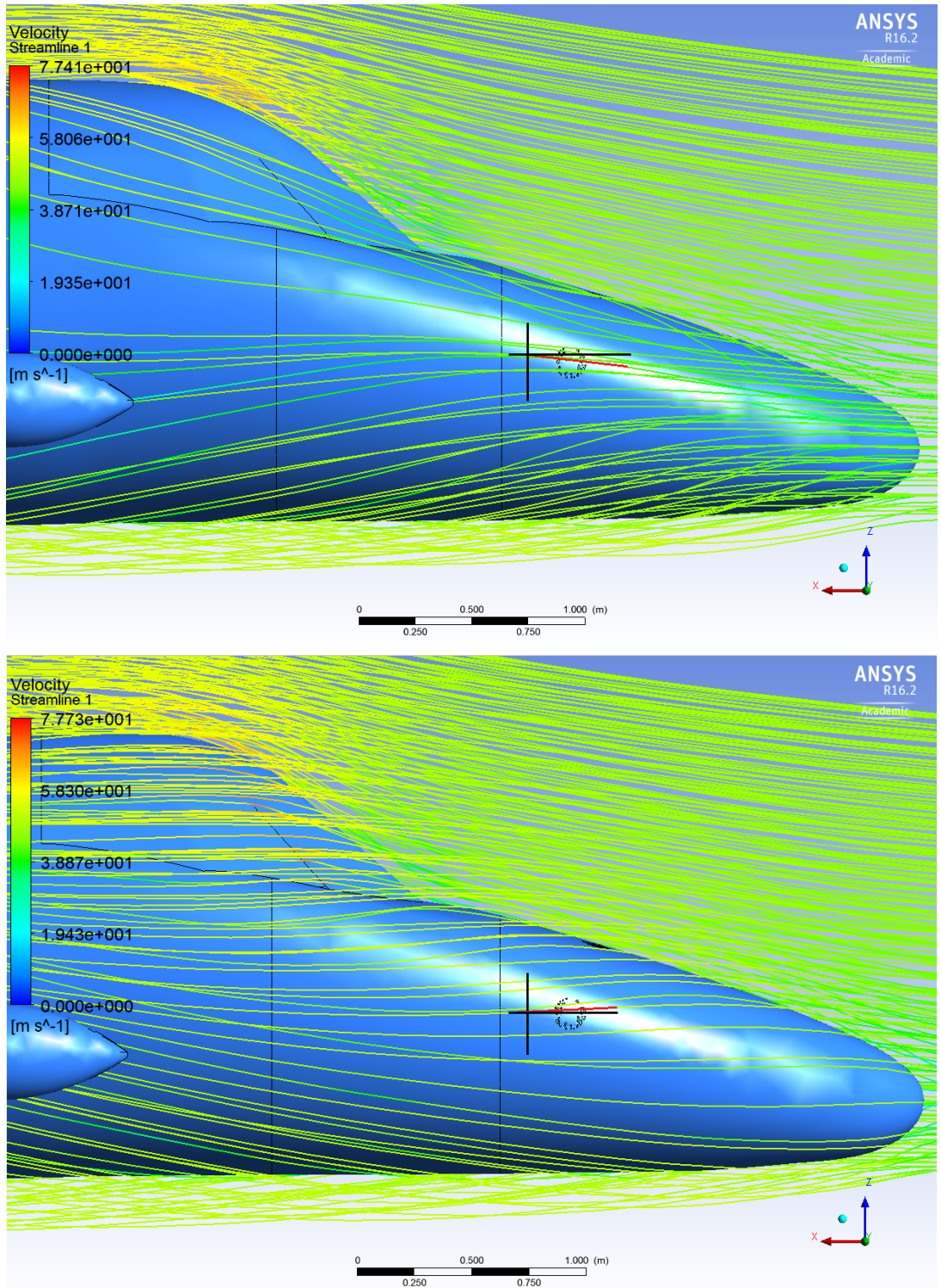

Figure 8: Effect of the nose on the local air flow direction around the angle of attack vane in the cases $\alpha=2.8^{\circ}, \beta=15^{\circ}$ upper and $\alpha=2.8^{\circ}, \beta=-15^{\circ}$ right. 


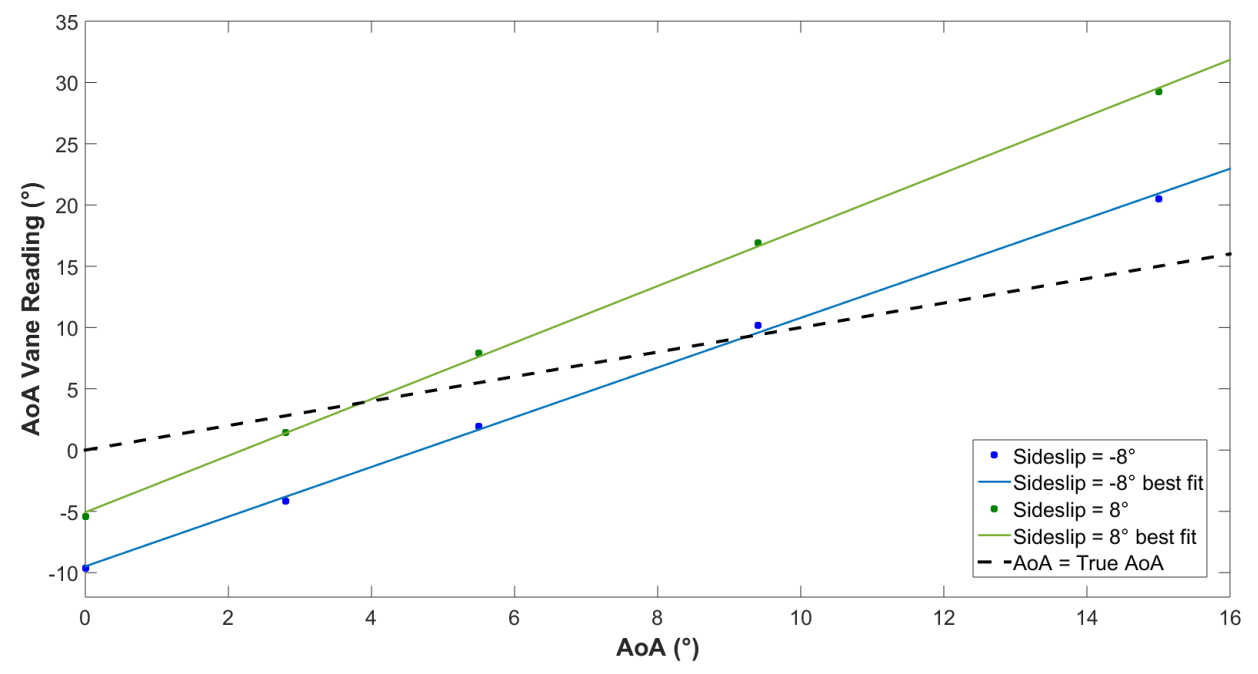

Figure 9: Difference in AoA vane reading depending on direction of sideslip. AoA vane in located on the leading side of the aircraft nose for sideslip angles $>0$.

vanes at intervals along the centre lines. This allowed an accurate prediction of the vanes position following it's exposure to the local flow to be made. Finally, the predicted flow angle at the vane locations was compared to the freestream angle of attack and sideslip.

The first observation to make is that since there is a single angle of attack vane positioned on the right-hand side of the nose, the accuracy of the readings from this vane will depend on it's exposure to the oncoming freestream. For positive sideslip angles the angle of attack vane is positioned on the leading side of the aircraft nose, but for negative sideslip angles the vane is obscured from the oncoming freestream by the aircraft nose. In the example shown in Fig 9 it is seen that the difference in angle of attack reading for $\pm 8^{\circ}$ sideslip angles ranges from $4^{\circ}$ for low angles of attack to $9^{\circ}$ for high angles of attack.

Furthermore, considering Fig 10 it is seen that the discrepancy between the angle of attack reading depending on a positive or negative sideslip angle increases as the sideslip angle increases. Fig 10 shows that when the vane is positioned on the leading side of the aircraft nose in the case of $2^{\circ}$ sideslip, the vane reading is not too dissimilar to that when the vane is on the obscured side, in the case of $-2^{\circ}$ sideslip. However, as the sideslip angle is increased to $\pm 15^{\circ}$ the discrepancy in angle of attack vane readings amplify.

Considering both Fig 9 and Fig 10 it is concluded that for low angles of attack, the vane reading is most accurate when the aircraft attains positive sideslip angles and for high angles of attack, the vane reading is most accurate when the aircraft attains negative sideslip angles. In general however, when the vane is positioned on the exposed side of the nose the vane will over-read the true angle of attack but when the vane is positioned on the obscured side the vane will under-read the true angle of attack. It can therefore be concluded that the calibration code which converts the raw vane position data in flight into a true angle of attack reading should depend on the sideslip angle. In the current set-up this is not the case, and hence some data may be incorrectly logged.

A second observation that can be made from the CFD simulations is that the accuracy of the sideslip vane readings also depends on the vanes position regarding the oncoming freestream. For both positive and negative sideslip angles, the corresponding leading vane yields the most 


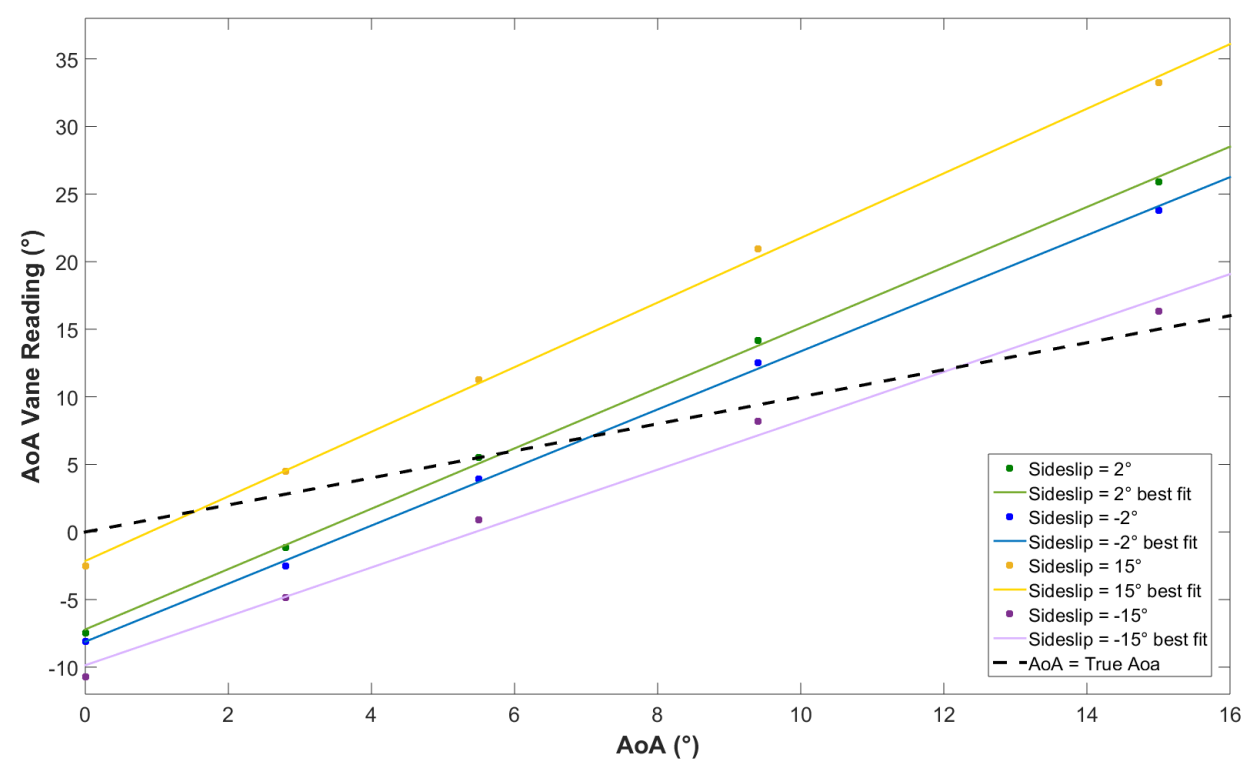

Figure 10: Difference in AoA vane reading depending on direction of sideslip. AoA vane in located on the leading side of the aircraft nose for sideslip angles $>0$.

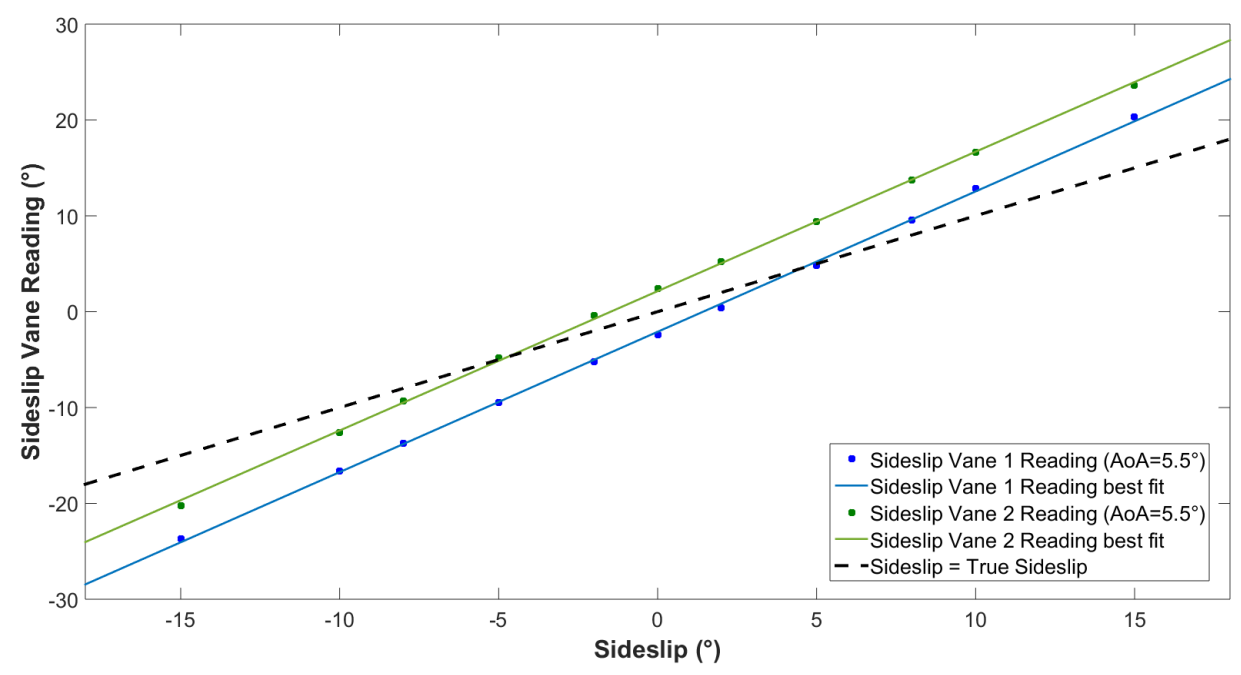

Figure 11: Difference in two sideslip vane readings for the case when $A o A=5.5^{\circ}$. Sideslip vane 1 is on the leading side of the nose for sideslip angles $>0$, and sideslip vane 2 is on the leading side of the nose for sideslip angles $<0$. 


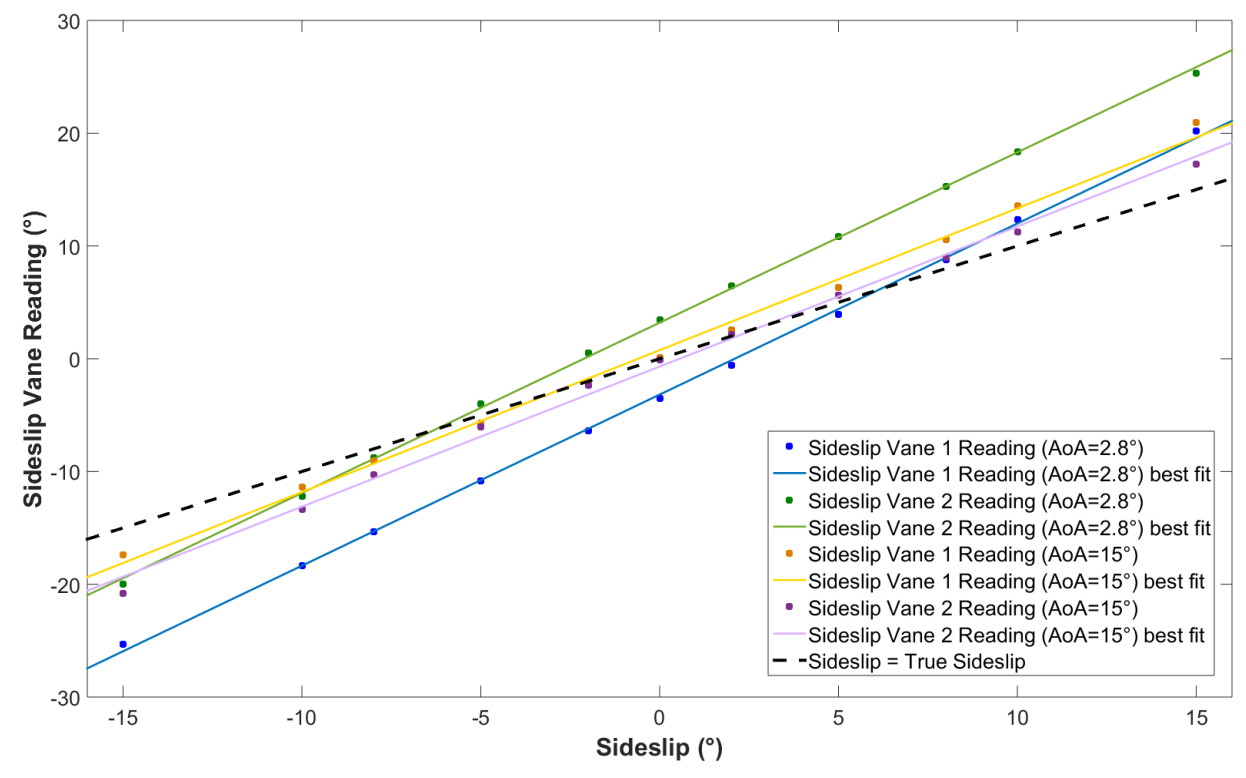

Figure 12: Difference in two sideslip vane readings for the cases $A \circ A=2.8^{\circ}$ and $A o A=15^{\circ}$. Sideslip vane 1 is on the leading side of the nose for sideslip angles $>0$, and sideslip vane 2 is on the leading side of the nose for sideslip angles $<0$.

accurate reading with regard to the true sideslip. Fig 11 shows the difference in sideslip vane readings for varying sideslip angles for the fixed case $\alpha=5.5^{\circ}$. It is seen that for negative sideslip angles vane number 2 attains the closest result to the true sideslip, and for positive sideslip angles vane number 1 attains the most accurate result.

The CFD results also reveal a greater difference between the two sideslip angle vane readings at lower angles of attack. In the example shown in Fig 12, the average discrepancy between the two vane readings at an angle of attack of $2.8^{\circ}$ is around $7^{\circ}$, but at an angle of attack of $15^{\circ}$ the discrepancy is significantly reduced to around $2^{\circ}$.

In the current aircraft data log the raw vane position data in flight is averaged to give a single sideslip angle, however the CFD results suggest that this calculation will only give an accurate reading when the sideslip angle is close to zero, see Fig 11 and Fig 12 . Therefore, the calibration equations for both angle of attack and sideslip vanes should be more definitively derived.

By means of multivariable regression the CFD results were analysed to determine the dependence of the angle of attack readings on the sideslip, and vice versa. All lines of best fit, such as the ones shown in Figs. 9, 10, 11, and 12, were studied to find the dependence of the gradient and $y$-intercept on the independent variable. Polynomials of increasing order were fitted to the data and the error of these curves analysed to determine the best fitting equations for the data. The following equations were found by the above method to correct the raw data readings from the flow angle vanes into the true angle of attack or sideslip,

$$
\alpha=\frac{\text { Raw AoA Reading }+0.0001888 \beta^{3}-0.007783 \beta^{2}-0.3006 \beta+7.7993}{0.00001754 \beta^{3}-0.000435 \beta^{2}+0.01541 \beta+2.1998}
$$




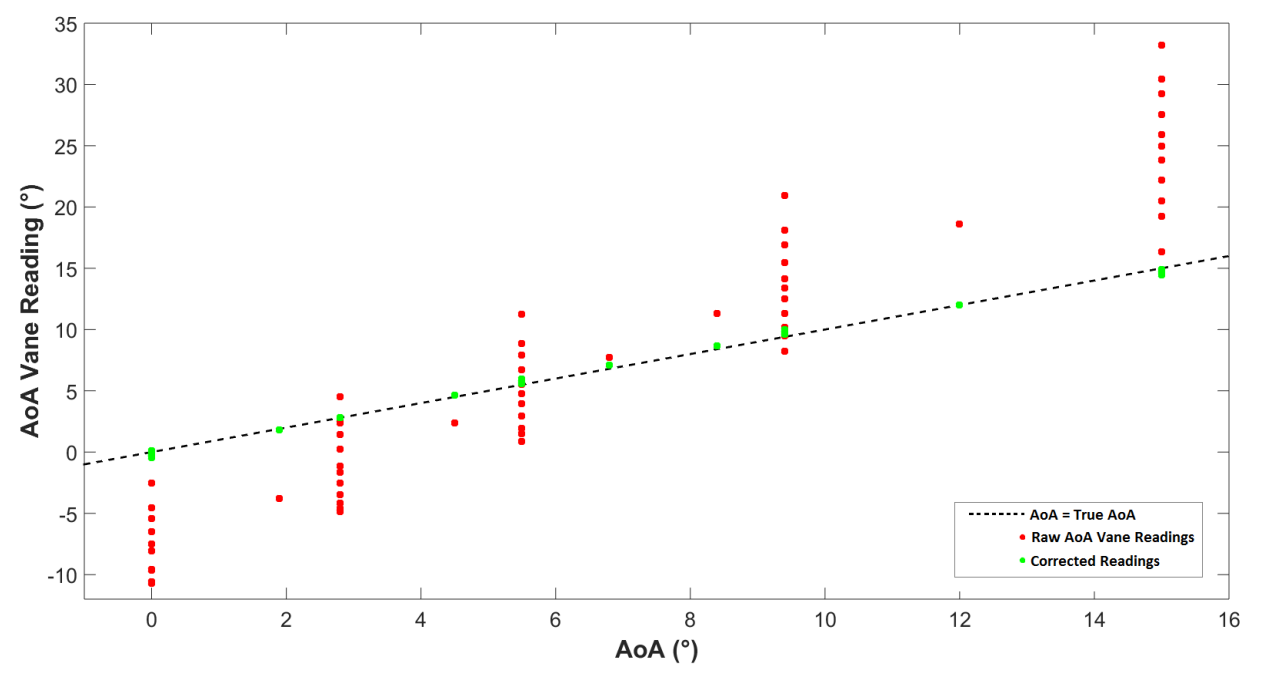

Figure 13: Equation (1) applied to the raw angle of attack vane data.

$$
\begin{aligned}
& \beta=\frac{\text { Raw SS1 Reading }-0.0000497 \alpha^{3}-0.00639 \alpha^{2}+0.449 \alpha-4.3769}{0.000196 \alpha^{2}+0.01774 \alpha-1.568}, \\
& \beta=\frac{\text { Raw SS2 Reading }+0.000416 \alpha^{3}-0.00302 \alpha^{2}-0.3786 \alpha+4.262}{-0.00000549 \alpha^{3}+0.0002635 \alpha^{2}+0.01876 \alpha-1.5647} .
\end{aligned}
$$

It can be seen from Figs, 13,14 , and 15 that applying the above equations to the raw flow angle data from the CFD simulations the data is corrected closely to the true angle of attack or sideslip, essentially mapping the inaccurate red points on to the green ones.

The obvious problem which arises when applying the above equations is that the true Angle of attack depends on the true sideslip and vice versa. An expression which relates the angle of attack to the raw sideslip vane readings can be found by equating equations (2) and (3), however an algebraic solution to the resulting sixth order polynomial is unobtainable via the Abel-Ruffini theorem.

However with little computational expense, a solution to the polynomial in the range $-15^{\circ}$ to $15^{\circ}$ can be found by implementing the Newton-Raphson method. The MATLAB code given in Appendix (B) shows how the method can be implemented to calculate the true angle of attack and sideslip if the raw vane readings are known. The CFD set-up angle of attack and sideslip angle are used to calculate the error of the Newton-Raphson method compared to the direct application of equations (1), (2) and (3), but in flight will be unknown. It is seen that the function typically takes less than 0.5 seconds to run and yields on average typical angle of attack and sideslip angle errors of $0.025^{\circ}$. Obviously the computation time depends on the speed of the on-board computer, but if the raw vane readings are directly read by the MATLAB function, realistically the true angle of attack and sideslip angle can be monitored at a rate of 3-4 times per second using this method. Due to the properties of the polynomial, for the range of raw vane readings typically experienced in flight conditions, a single root in the range $-15^{\circ}$ to $15^{\circ}$ is expected and hence modification of the initial conditions should not be required.

Table 5 summarizes the typical errors in the vane readings when the above calibration is 


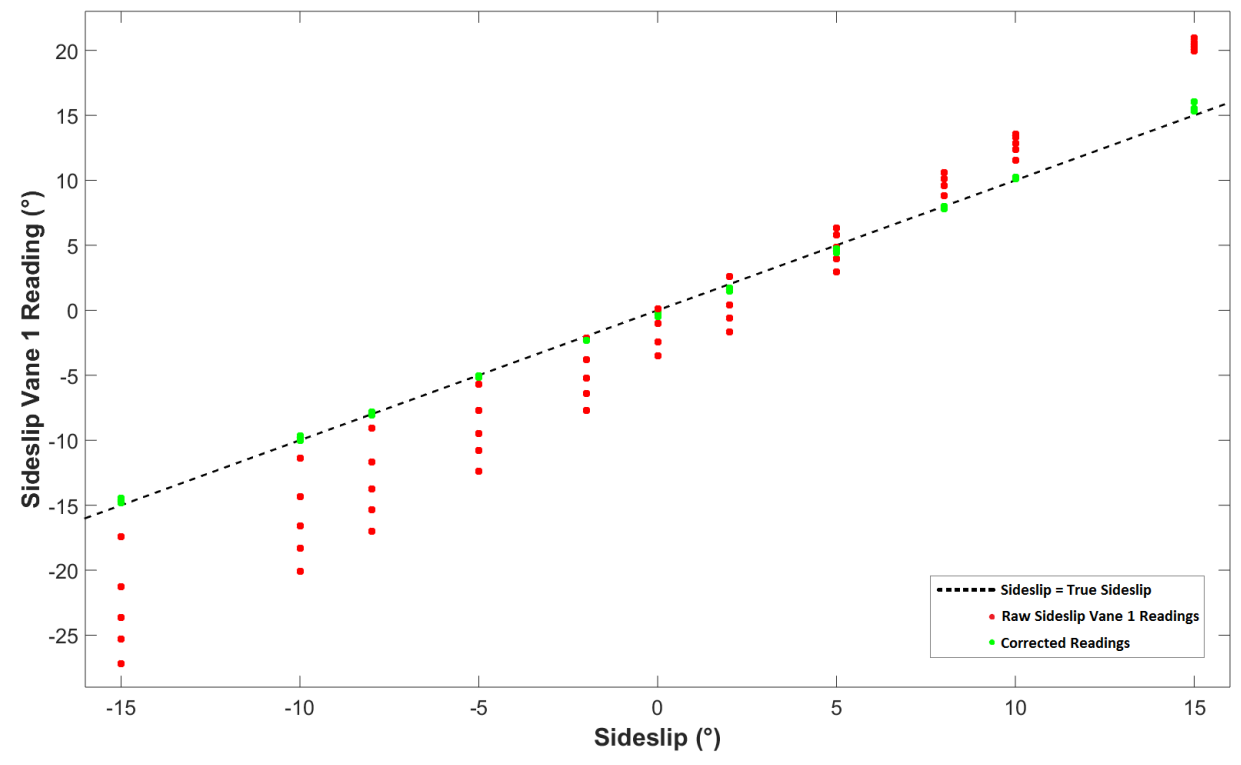

Figure 14: Equation 2, applied to the raw sideslip vane 1 data.

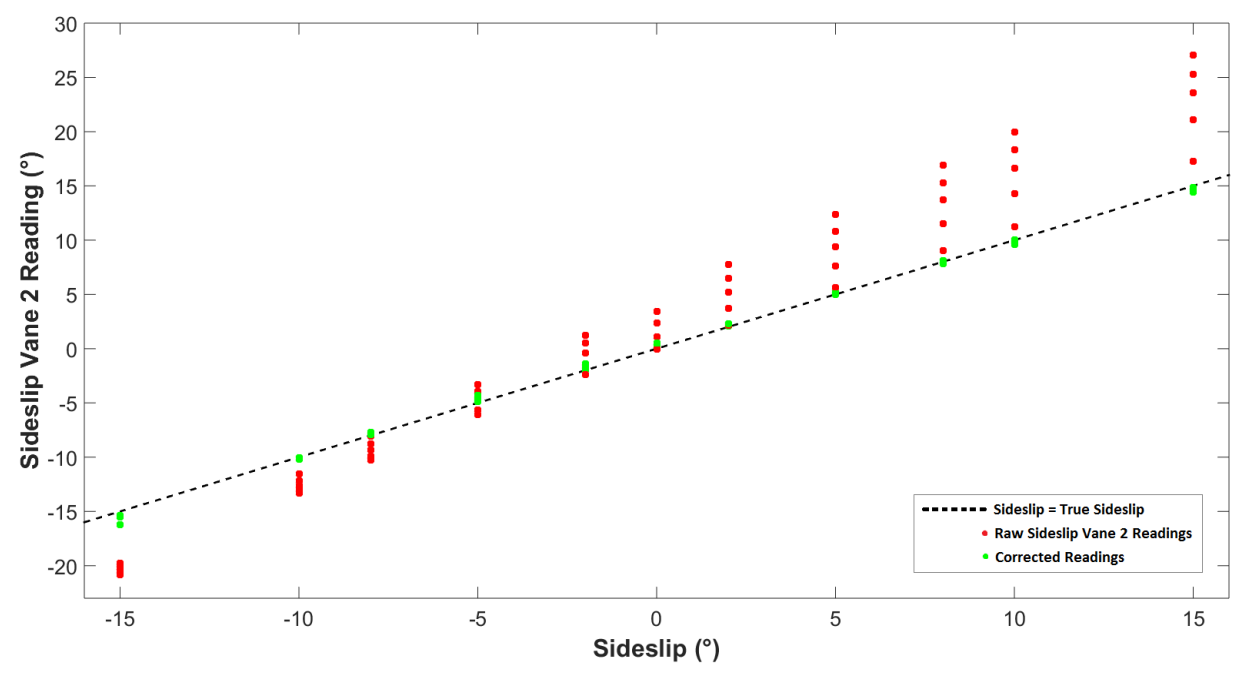

Figure 15: Equation (3) applied to the raw sideslip vane 2 data. 


\begin{tabular}{|l|c|}
\hline Ave. error of vane calibrations when compared to CFD input values (freestream) & Error $\left(^{\circ}\right)$ \\
\hline AoA error via equations (1), (2) \& 3) & 0.18 \\
Sideslip error via equations (1), (2) \& (3) & 0.24 \\
\hline AoA error via Newton's method solution to equations (1), (2) \& (3) & 0.89 \\
Sideslip error via Newton's method solution to equations (17), (2) \& (3) & 0.28 \\
\hline TOTAL AoA error & 0.90 \\
TOTAL Sideslip error & 0.36 \\
\hline
\end{tabular}

Table 5: Summary of vane calibration errors

applied. It is seen that if either the angle of attack or sideslip is a known parameter, the direct application of equations (1), (2) and (3) to find the other angle, yield accurate results to within a quarter of a degree for both angle of attack and sideslip. However, since in general both the angle of attack and sideslip angle will be unknown, the Newton-Raphson method will be implemented to solve the system of equations. Although the accuracy of the Newton-Raphson method can be increased by altering the criteria for convergence, the code given in Appendix (B) has been optimized so that an acceptable accuracy can be obtained quickly. This means that in flight, close to real-time readings are possible with an average error of less than $1^{\circ}$ for angle of attack and roughly a third of a degree for the sideslip angle.

\section{Flight Tests and Equipment Calibration}

In order to apply the theory developed from the CFD results outlined in the previous chapter to the Jetstream in flight, several flight tests and simple equipment calibrations are required.

Firstly, the relationship between the angle of attack and sideslip vane zero points with respect to the fuselage reference line used in the CFD simulations must be understood. A simple test was undertaken to confirm this difference. In the case of the angle of attack vane, an inclinometer with an accuracy of $\pm 0.1^{\circ}$ was placed on the vane and the readings were recorded for a number of points and compared to the raw output inside the aircraft cabin. It can be seen from Fig. 16 that the raw vane reading are approximately $9.1^{\circ}$ below the inclinometer readings with a $95 \%$ confidence interval of $\pm 2.006^{\circ}$. Therefore, the vane's zero position with respect to the fuselage reference line is $-9.1^{\circ}$ and hence this adjustment needs to be made to angle of attack readings for accurate comparison of CFD and flight test results. In the case of the sideslip vane, a simple laser test revealed that the zero point of both vanes is aligned accurately with the aircraft centreline, the CFD reference line, to within $0.5^{\circ}$. Hence, the raw sideslip vane readings require no reference line adjustment when used in the calibration.

Secondly, since the Jetstream is a twin turboprop aircraft where both propellers rotate anticlockwise (in the direction of flight) in any straight and level flight there must be a rudder input from the pilot to counteract the induced yaw from the propellers. This means that there will be an inherent sideslip in any straight and level flight, and this sideslip angle will depend on the airspeed. To get an idea of the relationship between the airspeed and the induced sideslip, the wealth of historic steady heading sideslip flight test data is analysed, where the data was collected using the previous flow angle calibration. For a given airspeed, the rudder angle ( $x$-axis) was plotted against the sideslip angle, and the $y$-intercept of the line of best fit in each case gave the inherent sideslip for a given airspeed. Fig. 17 shows the relationship between the airspeed and the propeller induced sideslip. Again, this relationship must be taken into account in order to make accurate comparisons between CFD and flight test results. As more data is collected using the 


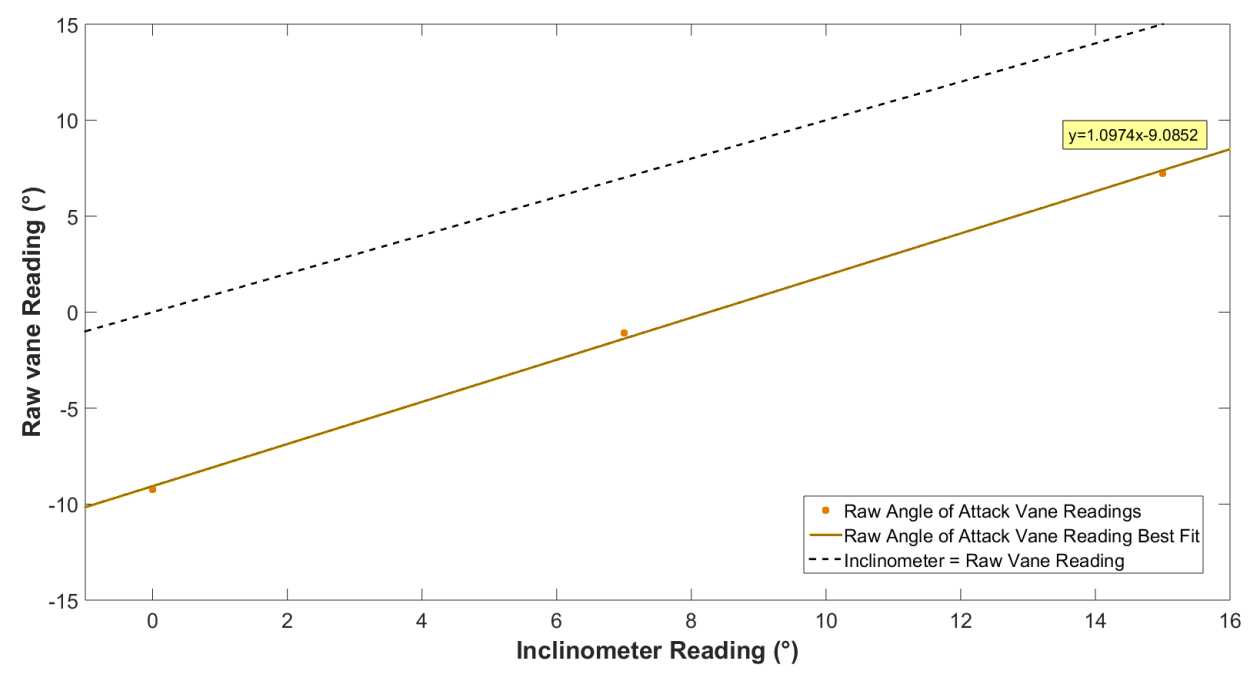

Figure 16: Graph to show the discrepancy in the angle of attack vane zero point and the fuselage reference line (CFD global reference).

calibration method proposed in this paper, an updated correction for propeller induced sideslip may be obtained.

Finally, as a backup for the proposed calibration for the vanes, the Inertial Reference System (IRS) mounted in the rear of the aircraft can be used. Since the CFD simulations use a reference line parallel to the fuselage, the relationship between this reference line and the IRS data is required for comparisons to flight test data. Pre-flight, a reference line inside the cockpit was found to be parallel to the fuselage reference. In flight, an inclinometer with an accuracy of $\pm 0.1^{\circ}$ was placed on the cockpit reference line and a series of straight and level, horizontal flight, conditions were flown at varying airspeeds. The data from the inclinometer was compared to the IRS data and it can be seen from the fitted line in Fig. 18 that the relationship is linear as expected but the IRS under-reads the angle of attack by the relationship shown in the panel in the top right of Fig. 18. This discrepancy must be taken into account when comparing in-flight angle of attack vane data with the true angle of attack. The angle of attack can be found from the IRS by taking the difference between the pitch angle and the flight path angle. Following the reference line correction, this data can be used in equations (2) and (3) to find the sideslip, followed by equation (1) to find the angle of attack. This method allows a comparison or check of the vane calibration via the IRS data, but should not be relied upon since the data from the IRS often exhibits significant scatter. For the specific case discussed here, the IRS data has a $95 \%$ confidence interval of $\pm 0.96^{\circ}$.

\section{Final Comparisons ANd Discussion}

Implementing the proposed calibration method and applying all corrections discussed in the previous section, it is possible to utilize the CFD data to directly compare the calibration method proposed in this paper with the current set-up on the Jetstream. For a full range of sideslip angles, it is seen that from Fig. 19, the new method more accurately predicts the true angle of attack and that the spread of results due to the sideslip angle variation is significantly reduced. The average error of angle of attack for the new calibration method is $\approx 0.25^{\circ}$ compared to $\approx 2.11^{\circ}$ 


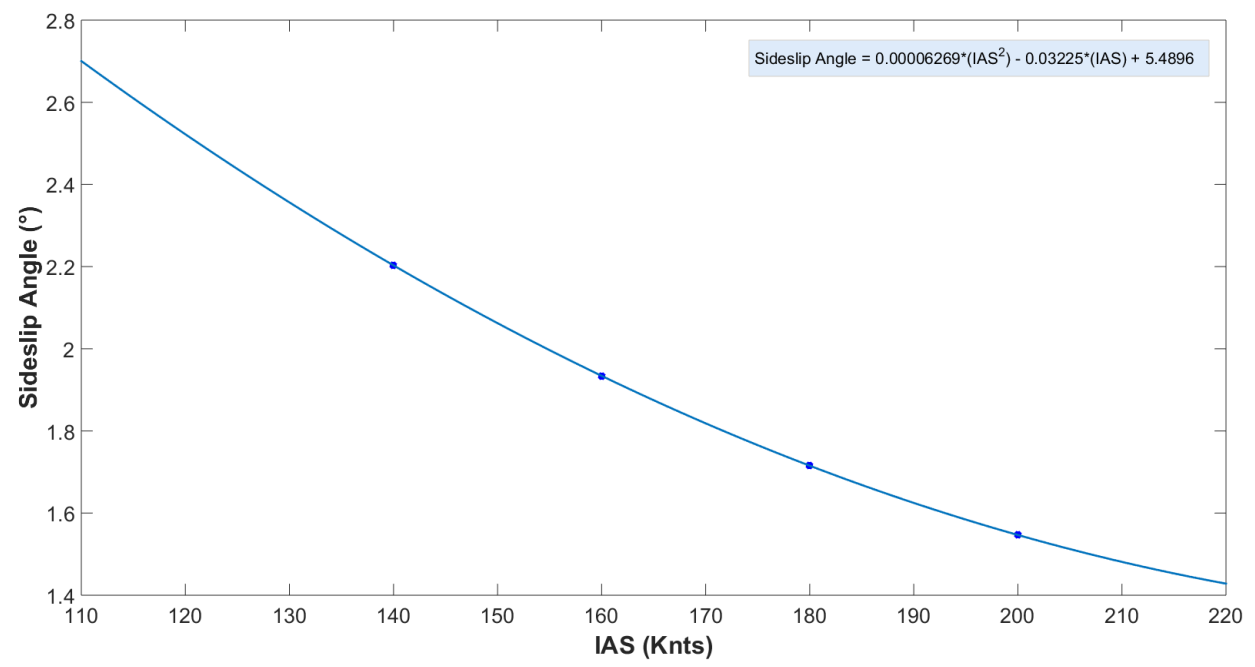

Figure 17: Graph showing the propeller induced sideslip at a given airspeed

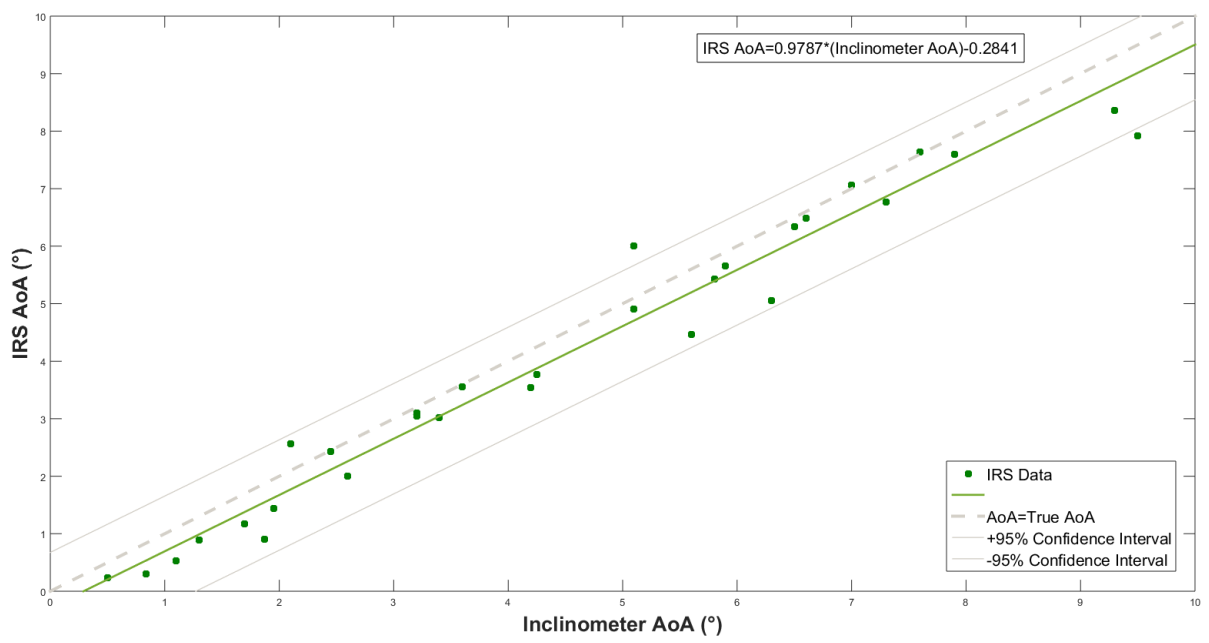

Figure 18: Graph to show the discrepancy in fuselage reference line (CFD global reference) and the aircraft IRS positioning. 


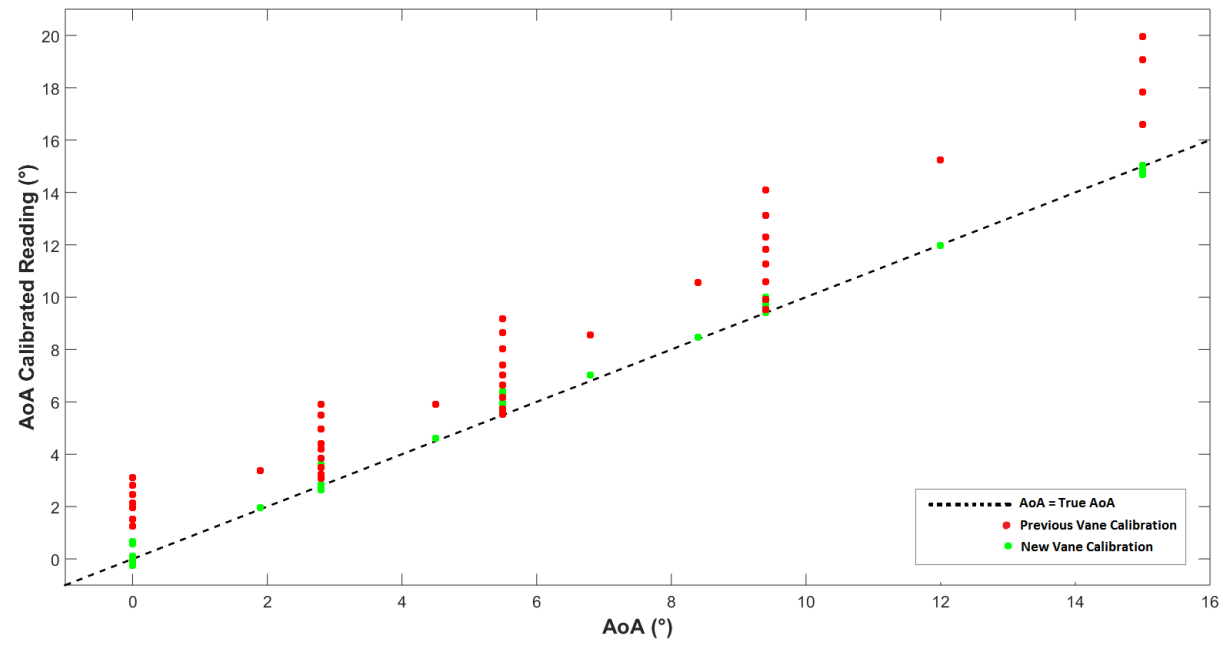

Figure 19: Comparison of the proposed calibration method with the current method for estimating the angle of attack.

for the old method. Similarly, it can be seen from Fig. 20 that for the entire angle of attack range considered, the corresponding sideslip measurements are also significantly more accurate. The average error of sideslip angle for the new calibration method is $\approx 0.10^{\circ}$ compared to $\approx 4.71^{\circ}$ for the old method.

Finally, the modified $C_{L}-\alpha$ curve is presented in Fig. 21 in comparison with the historic flight test data. The historic angle of attack data was collected using the IRS and so has been corrected using the adjustment discussed in the previous section, which was shown to have a 95\% confidence interval of less than $1^{\circ}$. Nevertheless, comparing this data with the CFD data it is clear that the short fall in lift coefficient may be accounted for by a number of external factors, most notably the propeller lift effects for which the relationship is expected to be linear. To confirm this, future flight tests should additionally record the vane readings using the proposed calibration method in order to make direct comparisons with the CFD analysis and also the current set-up on the aircraft.

\section{Conclusions}

The aim of this paper was to definitively understand the behaviour of the flow angle vanes on a BAe Jetstream 3102 for a full range of flight conditions to enable a more precise and reliable calibration of their readings.

The current vane calibration on the Jetstream calculates the 'true' angle of attack via a quadratic equation depending only on the angle of attack vane reading, and similarly for the sideslip angle. It seemed intuitive that, due to the positioning of the flow angle vanes on the nose of the aircraft, the deflection of airflow around the nose could potentially affect the vane readings in a much more complex way. A detailed CFD study was therefore undertaken to investigate this phenomena.

The results of the CFD simulations confirmed a significant dependency between the aircraft angle of attack, sideslip angle, and the flow angle vane readings. Using post processing techniques the local flow at the vane locations were analysed to predict the directional response of the flow angle vanes. Using a method of multivariable regression, a system of cubic equations were 


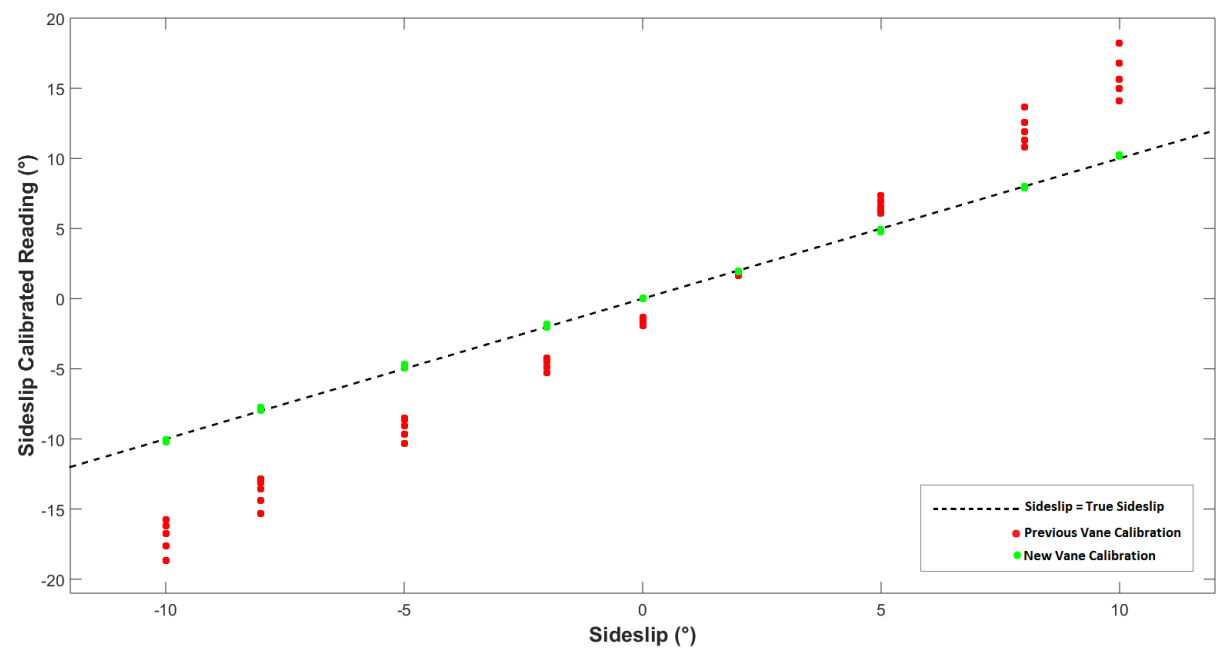

Figure 20: Comparison of the proposed calibration method with the current method for estimating the sideslip angle.

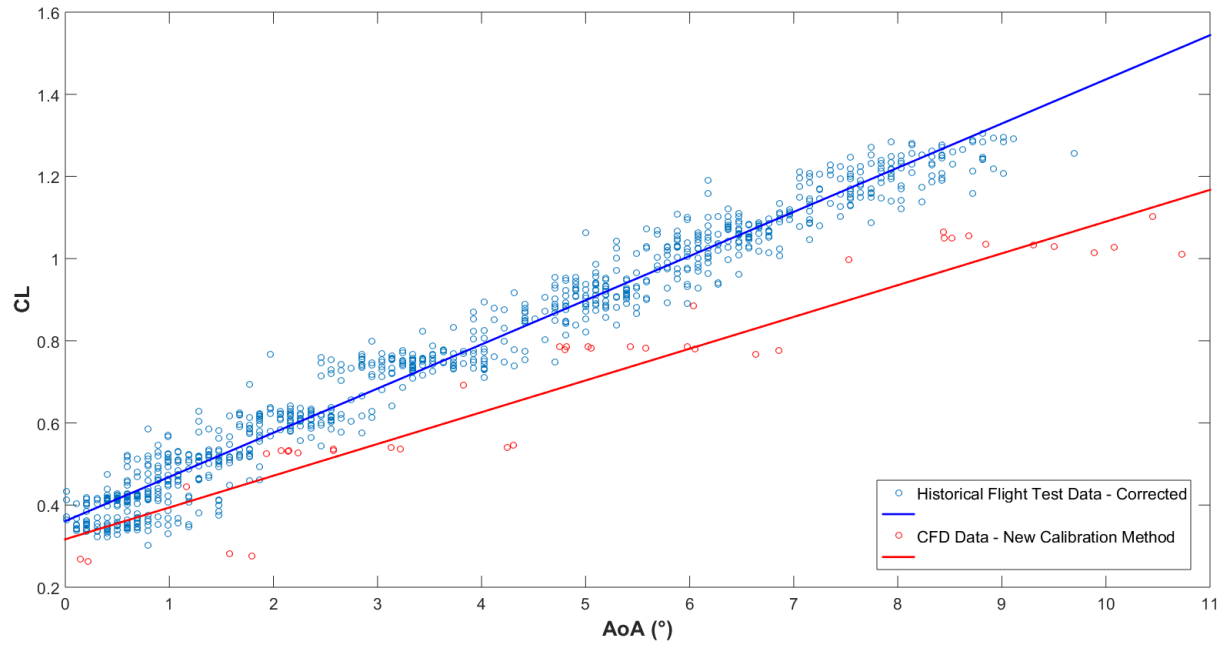

Figure 21: Comparison of the current and updated $C_{L}-\alpha$ curves. 
derived to describe this dependency and a solution strategy benefiting from Newton's method was implemented providing high accuracy.

Utilizing the proposed method, the error in angle of attack and sideslip angle were reduced from roughly $2.1^{\circ}$ to $0.25^{\circ}$, and $4.7^{\circ}$ to $0.1^{\circ}$ respectively.

Future work will aim to extend the research conducted here to include the effect of bank angle. 


\section{A. Appendix 1}

The standard direction cosine matrix $\mathbf{D}$, see [13] and [14], for a $(1,2,3)$ Euler angle sequence where the roll angle $\phi$ is applied first followed by the pitch angle $\theta$ and then the yaw angle $\varphi$, can be applied to any direction vector $(x, y, z)$,

$$
\mathbf{D}\left(\begin{array}{l}
x \\
y \\
z
\end{array}\right)=\left(\begin{array}{l}
\hat{x} \\
\hat{y} \\
\hat{z}
\end{array}\right)
$$

where

$$
\mathbf{D}=\left(\begin{array}{ccc}
\cos \theta \cos \varphi & \cos \theta \sin \varphi & -\sin \theta \\
\sin \theta \sin \phi \cos \varphi-\cos \phi \sin \varphi & \sin \phi \sin \theta \sin \varphi+\cos \phi \cos \varphi & \sin \phi \cos \theta \\
\cos \phi \sin \theta \cos \varphi+\sin \phi \sin \varphi & \cos \phi \sin \theta \sin \varphi-\sin \phi \cos \varphi & \cos \phi \cos \theta
\end{array}\right),
$$

and the vector $(\hat{x}, \hat{y}, \hat{z})$ may be found from a velocity vector using the identities

$$
\begin{aligned}
& \hat{x}=\frac{u}{V} \\
& \hat{y}=\frac{v}{V} \\
& \hat{z}=\frac{w}{V}
\end{aligned}
$$

where

$$
V=\sqrt{u^{2}+v^{2}+w^{2}}
$$

If a horizontal flight path is assumed, pitch angle corresponds to the angle of attack, yaw angle corresponds to the sideslip angle, and roll angle corresponds to the bank angle.

In the case of zero bank angle (ie. $\phi=0$ ), if the direction cosine matrix is applied to the direction vector $(1,0,0)$

$$
\begin{aligned}
& \hat{x}=\cos \theta \cos \varphi, \\
& \hat{y}=-\sin \varphi, \\
& \hat{z}=\sin \theta \cos \varphi .
\end{aligned}
$$

Therefore rearranging equation (A.4)

$$
\varphi=-\sin ^{-1}(\hat{y})
$$

Also, rearranging equation (A.5) and replacing into A.3

$$
\hat{x}=\frac{\cos \theta}{\sin \theta} \hat{z}
$$

and so

$$
\theta=\tan ^{-1}\left(\frac{\hat{z}}{\hat{x}}\right)
$$

Using the identities (A.1), equations (A.6) and A.7) can be rewritten in terms of the velocity components thus

$$
\begin{aligned}
\varphi & =-\sin ^{-1}\left(\frac{v}{V}\right) \\
\theta & =\tan ^{-1}\left(\frac{w}{u}\right)
\end{aligned}
$$


Similarly, in the case of zero angle of attack (ie. $\theta=0$ ), the direction cosine matrix applied to the direction vector $(1,0,0)$ gives

$$
\begin{aligned}
\varphi & =\cos ^{-1}\left(\frac{u}{V}\right) \\
\phi & =-\tan ^{-1}\left(\frac{w}{v}\right)
\end{aligned}
$$

and in the case of zero sideslip angle (ie. $\varphi=0$ ), the direction cosine matrix applied to the direction vector $(1,0,0)$ gives

$$
\begin{aligned}
\theta & =\cos ^{-1}\left(\frac{u}{V}\right) \\
\phi & =\tan ^{-1}\left(\frac{v}{w}\right)
\end{aligned}
$$




\section{B. Appendix 2}

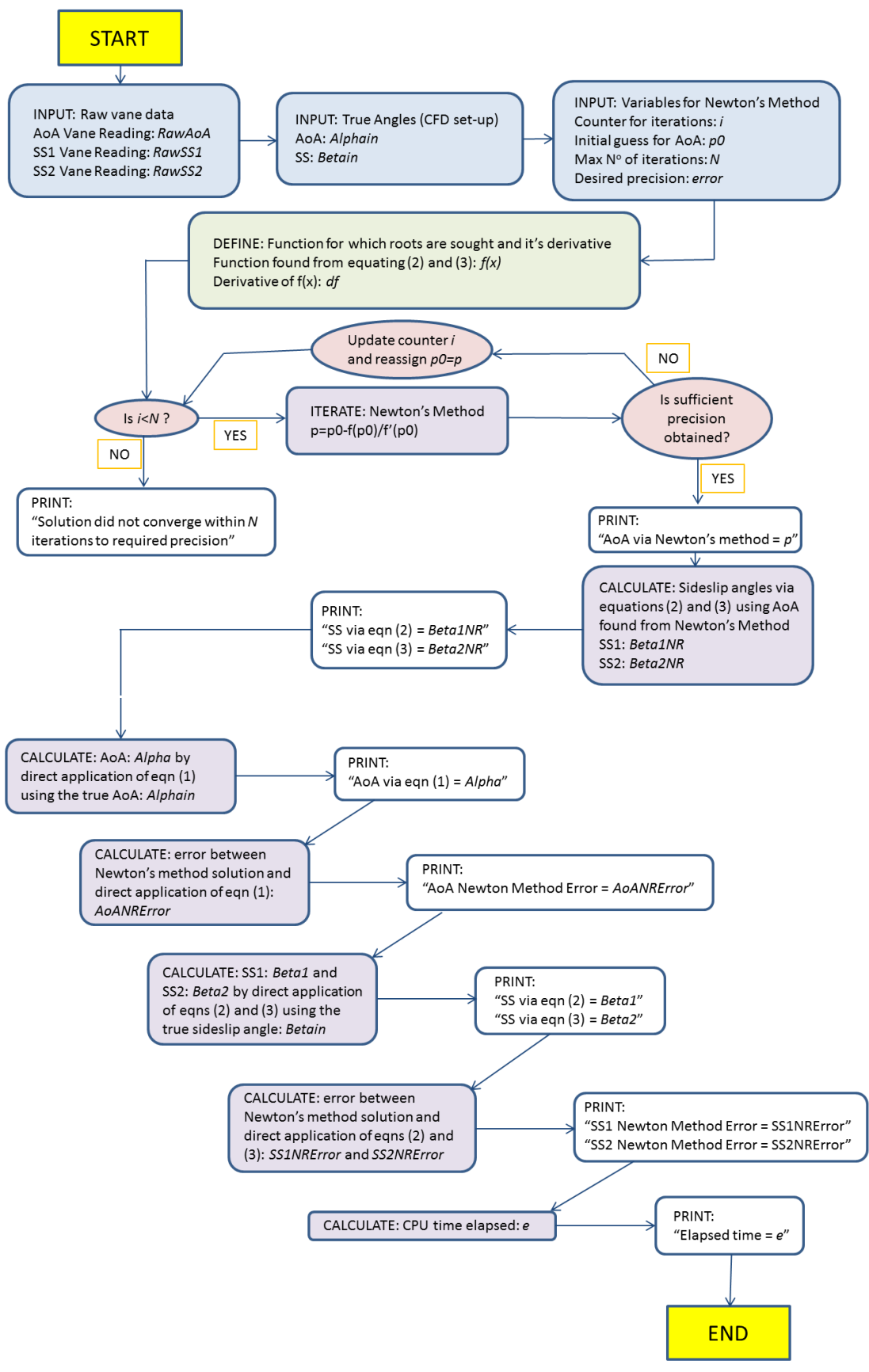

Figure B.1: Flowchart outlining the MATLAB code structure. Code variables are referred to in italics. 


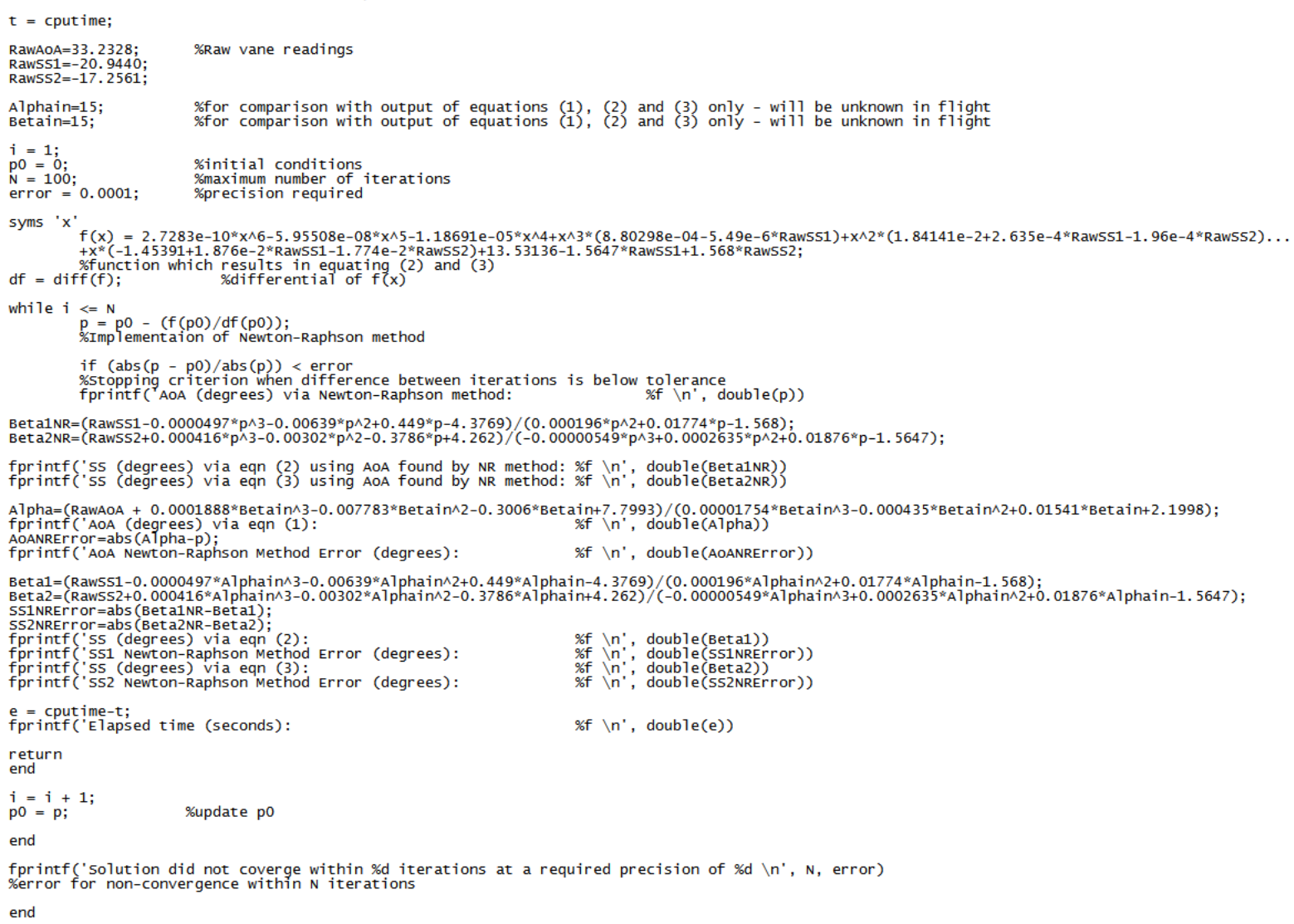

Figure B.2: MATLAB function calculating the AoA via Newton-Raphson method, followed by the sideslip angle via equations (2) and (3). 


\section{REFERENCES}

[1] J. E. Cashman, B. D. Kelly, and B. N. Nield. Operational Use of Angle of Attack on Modern Commercial Jet Airliners. AERO - Boeing Magazine - Flight Operations, (12), 2000.

[2] Y. Malinge (Ed.). Safety First - The Airbus Safety Magazine. (23), 2017.

[3] J. A. Lawford and K. R. Nippress. Calibration of air-data systems and flow direction sensors. Advisory Group for Aerospace Research and Development (AGARD) Flight Test Techniques Series, 1(AG-300), 1984.

[4] G. M Sakamoto. Aerodynamic charachteristics of a vane flowangularity sensor system capable of measuring flightpath accelerations for the mach number range from 0.40 to 2.54 . NASA Technical Note, (TN D-8242), 1976.

[5] P. F. Yaggy. A method for predicting the upwash angles induced at the propeller plane of a combination of bodies with an unswept wing. NACA Technical Note, (NACA-TN-2528), 1951.

[6] V. L. Rogallo. Effects of wing sweep on the upwash at the propeller planes of multi-engined airplanes. NACA Technical Note, (NACA-TN-2795), 1952.

[7] K. Rawlings III. A method for estimating upwash angle at noseboom-mounted vanes. Air Force Flight Test Centre, (AFFTC-TIM-81-1), 1981.

[8] C. Vessot. CFD Aerodynamic Model of the Jetstream 31. Master's thesis, Cranfield University, 2012.

[9] R. Parker. Investigation and Development of a 3D Non-Contact Scanning Process for CFD Model Generation. Master's thesis, Cranfield University, 2007.

[10] P. J. Roache. Verification and Validation in Computational Science and Engineering. Hermosa, New Mexico, USA, 1998.

[11] M. E. Breneckmann. Experimental Investigation of the Aerodynamics of a Wing in a Slipstream. Journal of the Aeronautical Sciences, 25(5):324-328, 1958.

[12] A. Jameson. The Analysis of Propeller-Wing Flow Interaction. NASA, 31:721-742, 1970.

[13] M. V. Cook. Flight Dynamics Principals - Second Edition. Elsevier, Oxford, UK, 2007.

[14] J. Diebel. Representing Attitude: Euler Angles, Unit Quaternions, and Rotation Vectors. Matrix, 58(15-16):1-35, 2006. 
2017-03-16

\section{CFD simulation of flow around angle of attack and sideslip angle vanes on a BAe Jetstream 3102 - Part 1}

Bennett, Christopher J.

Elsevier

C.J. Bennett, N.J. Lawson, J.E. Gautrey, A. Cooke, CFD simulation of flow around angle of attack and sideslip angle vanes on a BAe Jetstream 3102 - Part 1, Aerospace Science and Technology, Volume 68, September 2017, Pages 561-576 https://dspace.lib.cranfield.ac.uk/handle/1826/11616 Downloaded from Cranfield Library Services E-Repository 